\title{
Massive Vector Mesons and Gauge Theory
}

\author{
Michael Dütsch* \\ II Institut fuer Theoretische Physik \\ Universität Hamburg \\ Luruper Chaussee 149 \\ D-22761 Hamburg, Germany \\ email: duetsch@mail.desy.de \\ Bert Schroer \\ Institut für Theoretische Physik der FU-Berlin \\ presently: CBPF, Rua Dr. Xavier Sigaud, 150 \\ 22290-180 Rio de Janeiro, RJ, Brazil \\ email: schroer@cbpfsu1.cat.cbpf.br
}

June 1999

\begin{abstract}
We show that the requirements of renormalizability and physical consistency imposed on perturbative interactions of massive vector mesons fix the theory essentially uniquely. In particular physical consistency demands the presence of at least one additional physical degree of freedom which was not part of the originally required physical particle content. In its simplest realization (probably the only one) these are scalar fields as envisaged by Higgs but in the present formulation without the "symmetry-breaking Higgs condensate". The final result agrees precisely with the usual quantization of a classical gauge theory by means of the Higgs mechanism. But the emphasis is shifted: instead of invoking the gauge principle (and the Higgs
\end{abstract}

*Work supported by the Alexander von Humboldt Foundation and the Deutsche Forschungsgemeinschaft 
mechanism) on the local quantum field theory, the principles of local quantum physics restricted by the perturbative renormalizability demand "explains" (via Bohr's correspondence) the classical gauge principle as a selection principle among the many a priori (semi)classical possibilities of coupling vector fields among each other. Our method proves an old conjecture of Cornwall, Levin and Tiktopoulos stating that the renormalization and consistency requirements of spin $=1$ particles lead to the gauge theory structure (i.e. a kind of inverse of 't Hooft's famous renormalizability proof in quantized gauge theories) which was based on the on-shell unitarity of the $S$-matrix. Since all known methods of renormalized perturbation theory are off-shell, our proof is different and a bit more involved than the original arguments. We also speculate on a possible future ghostfree formulation which avoids "field coordinates" altogether and is expected to reconcile the on-shell S-matrix point of view with the off-shell field theory structure.

\section{Introduction}

In the development of understanding of the renormalization aspects for spin $=1$ interactions, the classical concepts of gauge and fibre bundles have played a crucial role. Without the closely related Higgs mechanism it would be hard to imagine, how in the stage of QFT at the end of the $60^{i e s}$, the incorporation of the electro-weak interaction into the framework of renormalizable field theory could have been achieved.

The main motivation for the present article starts from the remark that to our knowledge the Higgs mechanism via Higgs condensates within the setting of gauge theories has not a well-understood intrinsic quantum physical content. Hence we choose an alternative procedure by which we will construct the same physical results for interacting massive vector mesons in a quite different way which does not rely on the above concepts. In our approach based on the well-known real-time causal formulation of perturbative QFT, the renormalizability in the presence of selfinteracting vector mesons is the basic input requirement and the uniqueness and the gauge appearance of the polynomial interaction densities are consequences. With other words we would phrase 't Hooft's famous statement that gauge structure implies renormalizability the other way around: maintaining perturbative renormalizability in the presence of vector mesons explains the gauge structure. Since local quantum theory is more fundamental than classical, this 
brings interacting vector mesons into harmony with the properly understood Bohr's correspondence principle: it is the quantum theory which tells the classical which possibility among many couplings involving vector fields and lower spin fields it has to follow, namely the gauge invariant one.

We would not have gained much, and a cynic might claim that we have replaced one mystery (the gauge recipe) by another one (the renormalization prescription), but fortunately, we have some slightly more tangible results to offer. Our method brings into the open the long looming suspicion that the appearance of additional physical degrees of freedom (the alias Higgs particle but now without vacuum condensates) is a necessity, following from perturbative consistency up to second order (no claim outside of perturbation theory is made!)' In addition it suggests strongly that the physics of zero mass theory should be approached from massive vector mesons, the latter being conceptually (but not necessarily analytically) simpler. So as it happens often in physics, the new aspect does not so much lie in the physical results as such, but rather in the novel way in which they are obtained and in the interpretation associated with this derivation.

To avoid misunderstandings we emphasize that our approach supports the principle of local gauge invariance because we obtain the same results (Liealgebraic structure, necessity of the scalar "Higgs" field) by starting from quantum physical requirements instead of the conventional, semiclassical, differential geometric ideas. However it is also important to emphasize that crucial theoretical observations have been made in the first 5 years of existence of gauge theories (e.g. 't Hooft, Veltmann [45, 44] and Becchi, Rouet, Stora [3]). After almost 25 years there seems to be a consensus (based on the present theoretical aspects of the Standard model) that gauge theory, despite its impressive early successes, did not (yet) reach the level of maturity of other great theoretical developments in this century. Our present attempt at the physics of vector mesons outside (but not in contradiction to) gauge theory is very much motivated by this state of affairs. We consider this attempt as something preliminary. Although it starts from the very physical Wigner particle concept, it shares with the gauge approach the necessity of extension by unphysical degrees of freedom (ghosts), which in this case originates from renormalizability. This can be formulated as a cohomologi-

\footnotetext{
${ }^{1}$ Unfortunately this is not a structural theorem as e.g. the theorem connecting spontaneous symmetry-breaking with the appearance of "Goldstone modes" or the theorem connecting relating the appearance of "massive photons" with charge-screening; rather it still remains in the setting of renormalized perturbation theory [43].
} 
cal extension within the Wigner one particle spaces. The advantage of the present approach is that the auxiliary role of the ghosts as "renormalization catalyzers" "2 becomes more manifest. The reader finds some speculative remarks on where to look for a ghostfree formulation in the last sections.

Since some of our concepts are not new, it may be helpful to remind the reader of some historical precedents. Ever since theories in which vector mesons or higher spin particles became physically relevant in the late $50^{\text {ies }}$, there were two points of views to deal with such problem: either to start from the Wigner particle picture and stay close to particles and scattering theory, or to quantize classical gauge field theory (canonically or by functional integrals) and to make contact with (infra)particles at a later stage. In fact Sakurai, who introduced the term "Yang-Mills theory" [29], and who wanted to use quantized classical field theory for the description of massive vector mesons in strong interactions, encountered difficulties to reconcile the two points of view. In most of his contributions he therefore took a phenomenological non-geometric point of view within a dispersion theoretical setting. This was particularly advisable since, as a result of some criticism of Pauli, the use of the Yang-Mills model for the description of massive vector mesons was cast into question.

The discovery of the electro-weak theory and the renormalization of YangMills theories [44] led to a drastic change of that picture. The first approach, which tried to make the gauge principle for vector mesons more palatable from a particle physics point of view, found some protagonists in the early days of gauge theories (Lewellyn-Smith [26], Bell [4], Cornwall et al. [8]). It was argued that "tree unitarity" of the $S$-matrix implies that one can choose the fields such that the model describes a spontaneously broken gauge theory. Cornwall, Levin and Tiktopoulos even gave a heuristic argument that renormalizability requires tree unitarity. On the other side the gauge or Yang-Mills point of view enjoyed general popularity and became the predominant one, irrespective of whether it could be derived from a more particle dominated on-shell approach or not. Here we show that the tree unitarity viewpoint

\footnotetext{
${ }^{2}$ The ghosts are objects outside local quantum physics which are neither present in the original physical degrees of freedom (which one wants to couple) nor in the final physical cohomological subspace. But without their intermediate presence one does not know how to set up the renormalization machinery. In the perturbative framework we shall need fermionic and bosonic (scalar) ghost fields. The first correspond to the "Faddeev-Popov ghosts", the latter to the "Higgs ghosts" (which are also called "would be Goldstone bosons") or "Stückelberg fields".
} 
may be substituted by renormalizability within a suitable cohomologically extended Wigner particle setting. The presence of additional physical degrees of freedom (Higgs particles) follows from perturbative consistency, even though there is no concept of gauge symmetry breaking.

The main reason in favor of the gauge point of view was not only its success in describing problems of quantum matter coupled to external electromagnetic fields as well as its aesthetical mathematical appeal, but rather that it opened a path to a renormalizable theory of massive vector mesons, as it was demonstrated by 't Hooft and Veltman [45] [44]. This created, at least for some physicists, the impression that in addition to causality and spectral properties the general framework of QFT needs an additional principle for interacting massive vector mesons, namely the gauge principle. Here we propose a more intrinsic alternative framework which produces the same physical (gauge invariant) results solely on the principles of local quantum physics (LQP, for an introduction and overview see [21]) and perturbative renormalizability. It uses a simplified free form of the BRS formalism [3] for a cohomological extension of the Wigner representation theory as a mathematically less formal operator substitute for the Faddeev-Popov formalism [19] in functional representations. This special cohomological extension (which is only available for massive vector mesons!) lowers the operator dimension of free massive vector fields from their physical value 2 to the formal (classical) value 1 without loosing their point-like nature. Such a lowering is motivated by renormalizability within the framework of causal perturbation and the resulting theory is for all practical purposes equivalent to the gauge invariant Lagrangian approach[1. Our contention is that the renormalizability of interacting massive vector mesons alone uniquely fixes the theory, including its additional Higgs-like matter content. In other words the gauge and Higgs aspect from which one starts the Lagrangian quantization in the standard formulation is reduced to the concept of "renormalizability" with which we feel more comfortable because it occurs on the more fundamental level of local quantum physics where there is still a future chance for a profound understanding. The remaining intrinsic feature of the Higgs mechanism is the Schwinger charge screening caused by the Higgs-like matter degrees of

\footnotetext{
${ }^{3}$ Although most of the free fields which correspond to the $(\mathrm{m}, \mathrm{s})$ Wigner representation cannot be obtained in the setting of free Lagrangians $\mathcal{L}_{0}$, one can always rewrite a causal interaction $W$ in terms of Lagrangian field coordinatizations. This is necessary for path integral formulations, but not for the causal formulation of perturbations.

${ }^{4}$ Schwinger, in a little noticed paper and some more extensive published lecture notes
} 
freedom.

In more recent times a similar point of view has been taken up by Aste, Scharf and one of the authors (M.D.) [2]. We show however that their "free perturbative operator gauge invariance", which is a pure quantum formulation of gauge invariance, is not needed as an a priori perturbative requirement for pure massive theories: our physical consistency is a weaker requirement but fixes the theory to the same extent. Some aspects of our point of view appeared in the work of Grigore 20]. It also has been mentioned in the setting of Schwinger's work and of general quantum field theory by one of the authors (B. S) 33].

Recently there has been an approach to understand the local observable $*$-algebras of nonabelian gauge theories without addressing the particle content (which in the LQP framework is anyhow part of the separate more difficult representation theory associated with states on the algebra) [10. This approach was specially aimed at the zero mass theories with infrared problems, because the method does not require the existence of an adiabatic limit. In that case one cannot use the scattering theory of the physical particles and the BRS operators cannot be written as bilinear operators in free fields but they receive interacting contributions in every order. Therefore one has to face the more difficult problem of a changing position of the physical cohomology space inside the extended space depending on the perturbative order. The zero mass limit in our approach is conceptually and analytically complicated because it is expected to lead to charge liberation (the opposite of the Schwinger-Swieca charge screening) and the decoupling of the physical consistency (Higgs) degree of freedom. Those physical matter fields which will be charged in that limit cannot maintain their point-like localization, rather one expects that their localization cannot be better than semi infinite space-like (Mandelstam string-like). In fact the tight relation between these various phenomena generates the hope that by controlling those offshell infrared problems one may actually get an insight into this (even in

[38 thought about a massive phase in QED through the mechanism of charge screening but without (Higgs) vacuum condensates. In order to make his non-perturbative ideas of massive vector mesons (the Maxwellian interaction is renormalizable) more palatable, he invented the 2-dim. Schwinger model. (In fact in the Lowenstein-Swieca treatment of this model there is a chiral condensate (coming from the $\theta$-degeneracy), but after the dust has settled, the physical content is described in terms of a massive free field only). Schwinger's ideas were later elevated by Swieca into a theorem about vector mass generation via charge screening 43. 
perturbation theory) notoriously difficult localization structure.

For reasons of brevity we exemplify our procedure in a particular class, namely selfinteracting models of massive vector mesons. In section 2 we review the causal approach for massive vector mesons and its simplification as a result of the existence of a natural Fock reference space supplied by scattering theory. Our presentation uses a simplified quadratic BRS formalism. Physical consistency means that the $S$-matrix constructed on Fock space (which is the extended space, i.e. it includes ghost states) induces a well defined (unitary) operator on the physical "sub" space.

As a justification for the introduction of ghost fields we then describe in section 3 the apparent clash between renormalizability and the operator $\operatorname{dimension} \operatorname{dim} A=2$ of the free vector meson operators in the usual causal setting and its resolution via cohomological ghost extension, which can be preempted on the level of the Wigner one-particle spaces.

In the fourth section we formulate physical consistency in an alternative way which does not rely on scattering theory. The existence of the lowest dimensional interpolating physical (i.e. BRS-invariant) fields (the ones which we want to describe in our model as observable particles) does not only fix the form of these fields, but it also determines the interaction density (including the necessity of the alias Higgs particle) in agreement with sect. 2. In particular we find that the physical interpolating fields are composites in the elementary fields of the extended theory.

So the LSZ-type power series in terms of the physical incoming Fock space operators are modified (as we comment in sect. 5). This leads to a loss of the specific iterative law for the perturbative representation of the coefficient functions in terms of retarded products (for the fields). Only the general LSZ-like identification of coefficient functions of local fields involving multiple commutators of the local field with the incoming free field (generalized formfactors) remains valid [37]. The reason for this complication, which prevents the interchange of computations with the descend to the physical fields, is that the Wick-basis used for writing the latter in terms of linear combinations of composites (including ghosts) is not a natural basis for the physical fields (i.e. the fields which commute with the BRS charge $Q$ ).

Section 5 also contains some still speculative remarks of where one has to look for, if one wants to have a ghostfree formulation. In view of the fact that the previous sections have made clear that ghosts behave in some sense 
analogous to catalyzers 甲 in chemistry, this is not an academic problem but really goes to the root of understanding of renormalizability for higher spin where the standard causal approach breaks down. We are led to believe that such an approach must bypass the Bogoliubov-Shirkov transition operator $S(g)$ and be on-shell i.e. directly deal with the on-shell $S$-matrix and multi particle formfactors of physical fields.

We emphasize again that we consider the present formalism as transitory towards a completely ghostfree formulation of interactions involving higher spin particles. Although it does not achieve this aim, it creates a critical Bohr-Heisenberg attitude towards the many unobservable and formal aspects of the standard gauge formalism.

\section{Consistent perturbative construction of the $S$-matrix for massive vector fields}

The aim of this section is to construct the Stückelberg-Bogoliubov-Shirkov transition functional $S(\mathbf{g})$, which is the generating functional for the time ordered products of Wick polynomials. As most functional quantities this object is not directly observable but it gives rise to fundamental physical observables as the $S$-matrix, formfactors and correlation functions of observable fields. The notation should not be misread as the $S$-matrix by which we always mean the scattering operator computed with the LSZ or Haag-Ruelle scattering theory. Our model is that of selfinteracting massive vector mesons. Our procedure is related to the one of Scharf, Aste and the first author [2], 30], but similarly to a previous discussion by the other author [34 and to Grigore [20] as well as older articles as Lewellyn-Smith [26], we simply rely on physical consistency within the framework of local quantum physics and do not require such technical tools as "operator gauge invariance" although they tend to simplify calculations.

Using the Stückelberg-Bogoliubov-Shirkov-Epstein-Glaser method [5] [17]

\footnotetext{
${ }^{5}$ One introduces them in order to lower the operator dimension of the interaction density $W$ to the renormalizable value four. This is achieved by decreasing the dimension of the vector potential from two to one as a result of an unphysical cohomological extension of the Wigner one-particle representation theory. After the calculations have been done in the extended setting, one eliminates the ghosts by cohomological descend (BRS-invariance).
} 
we make the following perturbative Ansatz for $S(\mathbf{g})$

$S(\mathbf{g})=\mathbf{1}+\sum_{n=1}^{\infty} \frac{i^{n}}{n !} \int d^{4} x_{1} \ldots d^{4} x_{n} T_{j_{1} \ldots j_{n}}\left(x_{1}, \ldots, x_{n}\right) g_{j_{1}}\left(x_{1}\right) \ldots g_{j_{n}}\left(x_{n}\right), \quad \mathbf{g}=\left(g_{j}\right)_{j=0}^{G}$,

$g_{j} \in \mathcal{S}\left(\mathbf{R}^{4}\right)$, which is a formal power series in $\mathbf{g}$. The unknown $T_{j_{1} \ldots j_{n}}$ are operator valued distributions . They are constructed inductively by means of the following requirements (strongly influenced by the general Wightmanframework [4]):

(A) Specification of the model in first order: The first order expressions are the main input of the construction. They specify the model and must be local: $\left[T_{j}(x), T_{k}(y)\right]=0$ for $(x-y)^{2}<0$. We assume that $T_{0}(x) \equiv$ $W_{0}(x) \equiv W(x)=W(x)^{*}$ is the physically relevant interaction density in Fock space (i.e. a Poincaré covariant scalar composite described by a Wick polynomial). $\quad T_{j} \equiv W_{j}, j=1, \ldots, G$ are auxiliary interactions. The interaction $W_{j}$ is switched by the space-time dependent coupling "constant" $g_{j} \in \mathcal{S}\left(\mathbf{R}^{4}\right)$. The physically relevant $S$-matrix is obtained in the adiabatic limit: $g_{0} \rightarrow$ const., $g_{j} \rightarrow 0, j=1, \ldots, G$.

(B) Permutation symmetry: Due to the Ansatz (1) we may require permutation symmetry

$$
T_{j_{\pi 1 \ldots j_{\pi n}}}\left(x_{\pi 1}, \ldots, x_{\pi n}\right)=T_{j_{1} \ldots j_{n}}\left(x_{1}, \ldots, x_{n}\right), \quad \forall \pi \in \mathcal{S}_{n} .
$$

(C) Causality:

$S\left(\mathbf{g}^{(1)}+\mathbf{g}^{(2)}\right)=S\left(\mathbf{g}^{(1)}\right) S\left(\mathbf{g}^{(2)}\right) \quad$ if $\quad \cup_{j} \operatorname{supp} g_{j}^{(1)} \cap\left(\cup_{j} \operatorname{supp} g_{j}^{(2)}+\bar{V}_{-}\right)=\emptyset$,

where $\bar{V}_{\mp}$ denotes the closed backward/forward light cone. This requirement is equivalent to (see the appendix of [16]) 凹

$$
T_{j_{1} \ldots j_{n}}\left(x_{1}, \ldots, x_{n}\right)=T_{j_{1} \ldots j_{l}}\left(x_{1}, \ldots, x_{l}\right) T_{j_{l+1} \ldots j_{n}}\left(x_{l+1}, \ldots, x_{n}\right)
$$

\footnotetext{
${ }^{6}$ For questions concerning domains we refer to [17. For all operators which appear in sections 2 and 3 there exists a common dense invariant domain $\mathcal{D}$ and we restrict all operators to this subspace.

${ }^{7}$ The non-trivial part of this equivalence is that in the $n$-th order expression of (3) only special test functions appear, whereas (4) holds on $\mathcal{S}\left(\mathbf{R}^{4 n}\right)$.
} 


$$
\left\{x_{1}, \ldots, x_{l}\right\} \cap\left(\left\{x_{l+1}, \ldots, x_{n}\right\}+\bar{V}_{-}\right)=\emptyset .
$$

This means that $T_{j_{1} \ldots j_{n}}\left(x_{1}, \ldots, x_{n}\right)$ is a (well-defined) time ordered product of $W_{j_{1}}\left(x_{1}\right)$ $\ldots, W_{j_{n}}\left(x_{n}\right)$. Hence we use the notation

$$
T_{j_{1} \ldots j_{n}}\left(x_{1}, \ldots, x_{n}\right)=T\left(W_{j_{1}}\left(x_{1}\right) \ldots W_{j_{n}}\left(x_{n}\right)\right)
$$

Due to the induction with respect to the order $n$, the $T_{j_{1} \ldots j_{n}}$ are uniquely fixed by causality up to the total diagonal $\Delta_{n}=\left\{\left(x_{1}, \ldots, x_{n}\right) \mid x_{1}=x_{2}=\ldots=x_{n}\right\}$. The extension of the $T_{j_{1} \ldots j_{n}}$ to the total diagonal is non-unique. It is restricted by the following normalization conditions:

(D) Poincaré covariance;

(E) Unitarity: $S(\mathbf{g})^{-1}=S(\mathbf{g})^{*}$ for $\mathbf{g}=\left(g_{0}, 0, \ldots, 0\right), g_{0}$ real valued;

(F) Scaling degree: The degree of the singularity at the diagonal, measured in terms of Steinmann' scaling degree 40] 6] may not be increased by the extension. This ensures renormalizability by power counting if the scaling degree (or 'mass dimension') of all $W_{j}$ is $\leq 4$. This degree is a tool which is related to Weinberg's power counting.

Additional normalization conditions must be imposed, if one wants to maintain further symmetries or relations 9 , e.g. discrete symmetries (P,C,T), 'operator gauge invariance' (29-30) or the field equations of the interacting fields ((N4) in [10]), which can be obtained from the functional $S(\mathbf{g})$ (1) by Bogoliubovs formula (see sect.4).

The existence of the adiabatic limit restricts the extension additionally: for pure massive theories Epstein and Glaser [18] proved that, with correct mass and wave function (re)normalization the adiabatic limit of the functional $S(\mathbf{g})$ exists in the strong operator sense and it is this limit which we call 'S-matrix'. More precisely setting $\mathbf{g}_{\epsilon}(x):=\left(g_{0}(\epsilon x), 0, \ldots 0\right)$ the limit

$$
S_{n} \psi \equiv \lim _{\epsilon \rightarrow 0} S_{n}\left(\mathbf{g}_{\epsilon}\right) \psi
$$

\footnotetext{
${ }^{8}$ We adopt here the notion 'scaling degree' to operator valued distributions by using the strong operator topology. Note that the scaling degree of a Wick monomial agrees with its mass dimension.

${ }^{9}$ We consider symmetries and relations which are satisfied away from the total diagonal, due to the causal factorization (5) and the inductive assumption. Poincaré covariance (D) and unitarity (E) are of this type.
} 
exists $\forall \psi \in \mathcal{D}$, where $g_{0} \in \mathcal{S}\left(\mathbf{R}^{4}\right), g:=g_{0}(0)>0$ is the coupling constant and $S_{n}(\mathbf{g})\left(S_{n}\right.$ resp.) denotes the $n$-th order of the functional $S(\mathbf{g})(S$-matrix resp.). It follows that the $S$-matrix is unitary as an operator valued formal power series in Fock space [18]: $S=\sum_{n} S_{n}, S_{n} \sim g^{n}, S^{*} S=\mathbf{1}=S S^{*}$ on $\mathcal{D}$.

Due to this fact we solely consider models in which all fields are massive. In order that it makes physically sense to consider the $S$-matrix, we assume that there are no unstable physical particles as e.g. the W- and Z-bosons in the electroweak theory.

In vector meson theories (which are "gauge theories") the crucial problem is the elimination of the unphysical degrees of freedom. In the $S$-matrix framework this problem turns into the requirement that the $S$-matrix induces a well-defined unitary operator on the space of physical states (this is discussed in detail below). We will see that this condition is very restrictive: it determines the possible interactions to a large extent.

Let us first consider the free incoming fields. We quantize the free vector fields $\left(A_{a}^{\mu}\right)_{a=1, \ldots, M}$ by

$$
\left(\square+m_{a}^{2}\right) A_{a}^{\mu}=0, \quad\left[A_{a}^{\mu}(x), A_{b}^{\nu}(y)\right]=i g^{\mu \nu} \delta_{a b} \Delta_{m_{a}}(x-y), \quad A_{a}^{\mu *}=A_{a}^{\mu}
$$

(where $\Delta_{m}$ is the Pauli-Jordan distribution to the mass $m$ ), which corresponds to the "Feynman gauge". The representation of this *-algebra requires an indefinite inner product space. We, therefore, work in a Krein Fock space $\mathcal{F}$. We denote the scalar product by $(.,$.$) and A^{+}$is the adjoint of $A$ w.r.t. (.,.). Let $J$ be the Krein operator: $J^{2}=1, J^{+}=J$. Then the indefinite inner product $\langle.$, . $\rangle$ is defined by

$$
<a, b>\equiv(a, J b), \quad a, b \in \mathcal{F}
$$

and $*$ denotes the adjoint with respect to $<., .>$ :

$$
O^{*} \equiv J O^{+} J, \quad<O a, b>=<a, O^{*} b>
$$

Let $Q$ be an (unbounded) *-symmetrical nilpotent operator in $\mathcal{F}$

$$
Q=Q^{*} \quad(\text { on the dense invariant domain } \mathcal{D}), \quad Q^{2}=0
$$

By means of $Q^{2}=0$ one easily finds that $\mathcal{D}$ is the direct sum of three, pairwise orthogonal (w.r.t. (.,.)) subspaces [22] 223

$$
\mathcal{D}=\operatorname{ran} Q \oplus\left(\operatorname{ker} Q \cap \operatorname{ker} Q^{+}\right) \oplus \operatorname{ran} Q^{+}
$$


$\operatorname{ker} Q=\operatorname{ran} Q \oplus\left(\operatorname{ker} Q \cap \operatorname{ker} Q^{+}\right), \quad \operatorname{ker} Q^{+}=\operatorname{ran} Q^{+} \oplus\left(\operatorname{ker} Q \cap \operatorname{ker} Q^{+}\right)$

In addition we assume

$$
\left.J\right|_{\text {ker } Q \cap \text { ker } Q^{+}}=\mathbf{1} \quad \text { (positivity } \quad \text { assumption) }
$$

Then the $<., .>$-product is positive definite on

$$
\mathcal{H}_{\text {phys }} \equiv \operatorname{ker} Q \cap \operatorname{ker} Q^{+}
$$

and $\mathcal{H}_{\text {phys }}$ is interpreted as the physical subspace of $\mathcal{F}$. We denote the projectors on $\operatorname{ran} Q\left(\mathcal{H}_{\text {phys }}, \operatorname{ran} Q^{+}\right.$resp. $)$by $P_{-}\left(P_{0}, P_{+}\right.$resp. $)$

$$
\mathbf{1}=P_{-}+P_{0}+P_{+} \quad(\text { on } \mathcal{D})
$$

Note $Q=P_{-} Q P_{+}$. The positivity (13) and $Q=Q^{*}$ imply [23]

$$
P_{0}=P_{0} J P_{0}, \quad J=P_{0} J P_{0}+P_{-} J P_{+}+P_{+} J P_{-}
$$

and hence

$$
P_{0}^{*}=P_{0}, \quad P_{-}^{*}=P_{+}
$$

Let $S: \mathcal{D} \rightarrow \mathcal{D}$ be the (strong) adiabatic limit (6) of $S(g)$. We define

$$
S_{a b} \equiv P_{a} S P_{b}, \quad a, b \in\{-, 0,+\}
$$

and obtain

$$
P_{a} S^{*} P_{b}=\left(S_{(-b)(-a)}\right)^{*}
$$

For pedagogical reasons we introduce the matrix notation according to the decomposition (11)

$$
\begin{gathered}
J=\left(\begin{array}{ccc}
0 & 0 & P_{-} J P_{+} \\
0 & P_{0} & 0 \\
P_{+} J P_{-} & 0 & 0
\end{array}\right) \\
S=\left(\begin{array}{ccc}
S_{--} & S_{-0} & S_{-+} \\
S_{0-} & S_{00} & S_{0+} \\
S_{+-} & S_{+0} & S_{++}
\end{array}\right)
\end{gathered}
$$


and

$$
S^{*}=\left(\begin{array}{ccc}
\left(S_{++}\right)^{*} & \left(S_{0+}\right)^{*} & \left(S_{-+}\right)^{*} \\
\left(S_{+0}\right)^{*} & \left(S_{00}\right)^{*} & \left(S_{-0}\right)^{*} \\
\left(S_{+-}\right)^{*} & \left(S_{0-}\right)^{*} & \left(S_{--}\right)^{*}
\end{array}\right)
$$

by means of (19).

An alternative definition of the physical states is

$$
\mathcal{H}_{\text {phys }}^{\prime} \equiv \frac{\operatorname{ker} Q}{\operatorname{ran} Q}
$$

where the scalar product in $\mathcal{H}_{\text {phys }}^{\prime}$ is defined such that the map

$$
\mathcal{H}_{\text {phys }} \rightarrow \mathcal{H}_{\text {phys }}^{\prime}: \phi \rightarrow[\phi]
$$

is a pre Hilbert space isomorphism. ([ $\phi]$ denotes the equivalence class of $\phi$.) The first definition (i.e. $\mathcal{H}_{\text {phys }}$ ) has the advantage that the set of physical states is a subspace of the Krein Fock space $\mathcal{F}$, which has a clear particle interpretation. But $\mathcal{H}_{\text {phys }}$ is not Lorentz invariant (in contrast to $\mathcal{H}_{\text {phys }}^{\prime}$ ). The change of its position inside the total space under Lorentz-transformations is of course a result of the lack of Lorentz-invariance of J.

To describe free spin=1 fields the introduction of a BRST-formalism (as we just have done) is not necessary. However, our main topic is to describe the interacting theory in the adiabatic limit. So the incoming and outgoing fields are asymptotically free. Hence, being equipped with a characterization of the asymptotical physical states in terms of a BRST-formalism for free fields, our scattering point of view is much simplified.

Let $S^{*} S=\mathbf{1}=S S^{*}($ on $\mathcal{D})$. We now discuss two different formulations of the physical consistency of the $S$-matrix:

(i) A consistent $S$-matrix theory requires

$$
P_{0} S^{*} P_{0} S P_{0}=P_{0}=P_{0} S P_{0} S^{*} P_{0} \quad \Longleftrightarrow \quad\left(S_{00}\right)^{*}=\left(S_{00}\right)^{-1} \quad \text { on } \quad \mathcal{H}_{\text {phys }} \text {. }
$$

(ii) In the framework of the definition (22) of the physical states consistency means that $S$ and $S^{-1}=S^{*}$ induce well-defined operators on the factor space $\mathcal{H}_{\text {phys }}^{\prime}$ by the definition

$$
[\mathcal{O}][\phi] \equiv[\mathcal{O} \phi], \quad \mathcal{O}=S, S^{*}
$$


This holds true iff

$$
\mathcal{O} \operatorname{ker} Q \subset \operatorname{ker} Q \quad \wedge \quad \mathcal{O} \operatorname{ran} Q \subset \operatorname{ran} Q, \quad \mathcal{O}=S, S^{*}
$$

Due to $[\mathcal{O}]^{*}=\left[\mathcal{O}^{*}\right]$ the physical $S$-matrix $[S]$ is then unitary.

The following Lemma states that (i) is a truly weaker condition than (ii) and gives equivalent formulations of (ii).

Lemma 1: The following statements (a)-(g) are equivalent and they imply (h). But (h) does not imply the other statements if $Q \vDash 0$.

(a) $S S^{*}=\mathbf{1}=S^{*} S$ and $S \operatorname{ker} Q \subset \operatorname{ker} Q$ (i.e. $S_{+-}=0=S_{+0}$ ).

(b) $S S^{*}=\mathbf{1}=S^{*} S$ and $\left.[Q, S]\right|_{\operatorname{ker} Q}=0$.

(c) $S S^{*}=\mathbf{1}=S^{*} S$ and $S \operatorname{ran} Q \subset \operatorname{ran} Q$ (i.e. $S_{+-}=0=S_{0-}$ ).

(d) The matrix $S$ (21) has the form

$$
S=\left(\begin{array}{ccc}
S_{--} & S_{-0} & S_{-+} \\
0 & S_{00} & -S_{00}\left(S_{-0}\right)^{*}\left(S_{--}\right)^{*-1} \\
0 & 0 & \left(S_{--}\right)^{*-1}
\end{array}\right)
$$

where $S_{--}$and $S_{00}$ are invertible (on $\operatorname{ran} Q, \mathcal{H}_{\text {phys }}$ resp.) and $S_{00}, S_{--}, S_{-0}, S_{-+}$ satisfy

$$
\left(S_{00}\right)^{*}=\left(S_{00}\right)^{-1}, \quad S_{-+}\left(S_{--}\right)^{*}+S_{-0}\left(S_{-0}\right)^{*}+S_{--}\left(S_{-+}\right)^{*}=0 .
$$

(e) $S S^{*}=\mathbf{1}=S^{*} S$ and $S^{*} \operatorname{ker} Q \subset \operatorname{ker} Q\left(\right.$ i.e. $\left.\left(S_{+-}\right)^{*}=0=\left(S_{0_{-}}\right)^{*}\right)$.

(f) $S S^{*}=\mathbf{1}=S^{*} S$ and $\left.\left[Q, S^{*}\right]\right|_{\operatorname{ker} Q}=0$.

(g) $S S^{*}=\mathbf{1}=S^{*} S$ and $S^{*} \operatorname{ran} Q \subset \operatorname{ran} Q$ (i.e. $\left.\left(S_{+-}\right)^{*}=0=\left(S_{+0}\right)^{*}\right)$.

(h) $S S^{*}=\mathbf{1}=S^{*} S$ and $\left(S_{00}\right)^{*} S_{00}=P_{0}=S_{00}\left(S_{00}\right)^{*}$.

Proof: (b) $\Leftrightarrow$ (a) $\Leftrightarrow$ (g) and (f) $\Leftrightarrow$ (e) $\Leftrightarrow$ (c) hold trivially true.

(a) $\Leftrightarrow$ (e): (a) implies $S_{k} \operatorname{ker} Q \subset \operatorname{ker} Q$ for each order $S_{k}$ of $S$. Therefore,

$$
\left(S^{*}\right)_{n}=\left(S^{-1}\right)_{n}=\sum_{r=1}^{n}(-1)^{r} \sum_{n_{1}, \ldots, n_{r} \geq 1, n_{1}+\ldots+n_{r}=n} S_{n_{1} \ldots S_{n_{r}}}
$$

maps $\operatorname{ker} Q$ in $\operatorname{ker} Q$ and, hence, this holds also true for $S^{*}=\sum_{n}\left(S^{*}\right)_{n}$. (e) $\Rightarrow$ (a) follows analogously.

$(\mathrm{a}),(\mathrm{c}) \Leftrightarrow(\mathrm{d})$ : By a straightforward calculation one verifies that the equations

$S_{+-}=0, S_{+0}=0, S_{0-}=0 \quad$ and $\quad \sum_{c}\left(S_{(-c)(-a)}\right)^{*} S_{c b}=\delta_{a b} P_{a}=\sum_{c} S_{a c}\left(S_{(-b)(-c)}\right)^{*}$ 
are equivalent to $(\mathrm{d})$.

(a), (c) $\Rightarrow(\mathrm{h})$ : Choosing $a=0=b$ in (27) we obtain $\left(S_{00}\right)^{*} S_{00}=P_{0}=$ $S_{00}\left(S_{00}\right)^{*}$.

To show that $(\mathrm{h})$ does not imply the other statements for $Q \quad=0(\Leftrightarrow$ $\operatorname{ran} Q \vDash 0 \Leftrightarrow \operatorname{ran} Q^{+} \vDash 0$ ), we give two examples for $S$ which satisfy (h) but not $(\mathrm{d})$ :

- The condition (h) is invariant under an exchange of $Q$ and $Q^{+}$. Therefore, there exists a solution $\mathrm{S}$ of $(\mathrm{h})$ which maps $\operatorname{ker} Q^{+}$in $\operatorname{ker} Q^{+}$(and/or $\operatorname{ran} Q^{+}$in $\operatorname{ran} Q^{+}$), i.e. $S$ is a lower triangular matrix.

- The following $S$-matrix fulfills $(\mathrm{h})$ and $S_{+-} \vDash 0 \vDash S_{-+}$(if $c d \models 0$ ):

$$
S=\left(\begin{array}{ccc}
a e^{i \alpha} P_{-} & 0 & i c e^{i \alpha} P_{-} J P_{+} \\
0 & e^{i \phi} P_{0} & 0 \\
i d e^{i \alpha} P_{+} J P_{-} & 0 & b e^{i \alpha} P_{+}
\end{array}\right)
$$

with $a, b, c, d \in \mathbf{R}, \quad \alpha, \phi \in \mathbf{R}$ and $a b+c d=1$.

In references [2], [30] physical consistency of the $S$-matrix is satisfied by requiring a perturbative condition which implies $[Q, S]=0$, namely the

'free perturbative operator gauge invariance' $\square$ (or ' $Q$-divergence condition'): Let $W \equiv T_{0}$ be the interaction Lagrangian. Then there exists a Wick polynomial $W_{1}^{\nu}$ with

$$
[Q, W(x)]=i \partial_{\nu} W_{1}^{\nu}(x)
$$

and the time ordered products of $W$ and $W_{1}^{\nu}$ fulfil

$$
\left[Q, T\left(W\left(x_{1}\right) \ldots W\left(x_{n}\right)\right)\right]=i \sum_{l=1}^{n} \partial_{\mu}^{x_{l}} T\left(W\left(x_{1}\right) \ldots W_{1}^{\mu}\left(x_{l}\right) \ldots W\left(x_{n}\right)\right)
$$

Let us assume that (30) holds true to all orders $\leq(n-1)$. Due to the causal factorization (1) the requirement (30) is then satisfied away from the total diagonal, i.e. on $\mathcal{S}\left(\mathbf{R}^{4 n} \backslash \Delta_{n}\right)$. Hence, (30) is an additional normalization

\footnotetext{
${ }^{10}$ The reason for this name stems from the fact that $Q$ is the generator of the BRSTtransformation of the free incoming fields. For the present purpose we will simply refer to it as the "Q-divergence condition" for time ordered products since it has nothing to do with the classical notion of gauge invariance in the differential geometric setting of fibre bundles, but secures that the true $S$-matrix is physical. Roughly speaking it is the off shell version of physicality of the on-shell $S$-matrix.
} 
condition for $T(W \ldots W)$ and $T\left(W \ldots W_{1}^{\mu} \ldots W\right)$. But it is a highly non-trivial task to prove that there exists an extension to the diagonal which satisfies (30) and the other normalization conditions (D), (E) and (F).

In contrast to $[Q, S]=0$ or $\left.[Q, S]\right|_{\operatorname{ker} Q}=0$, the $Q$-divergence condition (29) makes sense also in models in which the adiabatic limit does not exist, e.g. for massless selfinteracting vector fields ("nonabelian gauge theories"). For massless $S U(N)$-Yang-Mills theories it has been proved that the $Q$-divergence condition (29-30) (more precisely the corresponding C-number identities which imply (29-30) ) can be satisfied to all orders [12], [13], and that these C-number identities imply the usual Slavnov-Taylor identities [14]. In addition, (29-30) determines to a large extent the possible structure of the model (see below). We emphasize that this is a pure quantum formulation of gauge invariance, without reference to classical physics.

In the present case of pure massive vector mesons (or more generally in "gauge" theories in which the strong adiabatic limit (6) of the transition functional $S(\mathbf{g})$ exists) we proceed in an alternative way. We do not require the $Q$-divergence condition (29-30 as a new physical principle, instead we simply require physical consistency of the $S$-matrix, which means

$$
\left.[Q, S]\right|_{\operatorname{ker} Q}=0
$$

by Lemma 1. This is a weaker condition than (29-30). But we will see that it determines the theory to the same extent. Our procedure is similar to Grigore 20. [1]

First we construct the operator $Q$ (10) which defines the physical states. For massless vector meson theories the procedure is well-known [25], [11]. The nilpotency of $Q$ gives reason to introduce an anticommuting pair of ghost

\footnotetext{
${ }^{11}$ However, there are two conceptual shortcomings in these papers. It is overlooked that most of the trilinear terms in $W$ vanish in the adiabatic limit due to energy-momentum conservation and, hence, to first order the condition (31) yields no information about the trilinear terms in $W$ (see appendix A).

By using the terminology introduced below the second shortcoming can be described as follows: the Higgs field(s) is/are treated as scalar partner(s) (with arbitrary mass $m_{H} \geq 0$ ) of the massless vector field(s), which does/do not appear in $Q$ and, hence, is/are physical. By chance this works for the electroweak theory (there is one massless vector field and one Higgs field is needed). But e.g. in the present case of pure massive vector mesons, there would be no Higgs field and such a model is physically inconsistent (31) to second order. This insufficiency does not appear in [2] and [30].
} 
fields $u_{a}, \tilde{u}_{a}$ for each vector field $A_{a}$ (fermionic ghosts). Then we define

$$
Q \equiv \int d^{3} x \sum_{a} \partial_{\nu} A_{a}^{\nu}(x) \overleftrightarrow{\partial_{0}} u_{a}(x)
$$

Turning to massive vector fields $A_{a}$ (17) we give the fermionic ghost fields the same masses $m_{a}$ (otherwise the current $\sum_{a} \partial_{\nu} A_{a}^{\nu} \overleftrightarrow{\partial}_{\mu} u_{a}$ or $\sum_{a}\left(\partial_{\nu} A_{a}^{\nu}+\right.$ $\left.m_{a} \phi_{a}\right) \overleftrightarrow{\partial}_{\mu} u_{a}$ (see below) would not be conserved)

$$
\begin{gathered}
\left(\square+m_{a}^{2}\right) u_{a}=0, \quad\left(\square+m_{a}^{2}\right) \tilde{u}_{a}=0, \quad\left\{u_{a}(x), u_{b}(y)\right\}=0, \quad\left\{\tilde{u}_{a}(x), \tilde{u}_{b}(y)\right\}=0, \\
\left\{u_{a}(x), \tilde{u}_{b}(y)\right\}=-i \delta_{a b} \Delta_{m_{a}}(x-y), \quad u_{a}^{*}=u_{a}, \quad \tilde{u}_{a}^{*}=-\tilde{u}_{a} .
\end{gathered}
$$

If we insert the massive $A_{a}$ and $u_{a}$ fields into the formula (32) for $Q$ the nilpotency is lost

$2 Q_{\text {naive }}^{2}=\left\{Q_{\text {naive }}, Q_{\text {naive }}\right\}=\int d^{3} x \int d^{3} y\left[\partial_{\mu} A_{a}^{\mu}(x), \partial_{\nu} A_{b}^{\nu}(y)\right] \overleftrightarrow{\partial}_{x_{0}} \overleftrightarrow{\partial}_{y^{0}} u_{a}(x) u_{b}(y) \Leftarrow 0$

To restore the nilpotency we proceed as follows [23]: to each vector field $A_{a}$ we consider a scalar field $\phi_{a}$ with the same mass $m_{a}$

$$
\left(\square+m_{a}^{2}\right) \phi_{a}=0, \quad\left[\phi_{a}(x), \phi_{b}(y)\right]=-i \delta_{a b} \Delta_{m_{a}}(x-y), \quad \phi_{a}^{*}=\phi_{a} .
$$

We call the fields $\phi_{a}$ also 'ghost fields' (bosonic ghosts) because they are unphysical (see below). Then, due to $\left[\partial_{\mu} A_{a}^{\mu}+m_{a} \phi_{a}, \partial_{\nu} A_{b}^{\nu}(y)+m_{b} \phi_{b}\right]=0$, the charge

$$
Q \equiv \int d^{3} x \sum_{a}\left(\partial_{\nu} A_{a}^{\nu}(x)+m_{a} \phi_{a}(x)\right) \overleftrightarrow{\partial}_{0} u_{a}(x)
$$

is nilpotent and symmetrical. Later we shall see that an additional scalar field $H$ with arbitrary mass $m_{H} \geq 0$ is needed ${ }^{\mathbb{3} 3}$

$$
\left(\square+m_{H}^{2}\right) H=0, \quad[H(x), H(y)]=-i \Delta_{m_{H}}(x-y), \quad H^{*}=H .
$$

\footnotetext{
${ }^{12}$ The convergence of this integral (and also of the corresponding expression (36) in the massive theory) can be shown by using a method of Requardt 28, 10.

${ }^{13}$ For simplicity we only consider models in which one additional scalar field suffices for a consistent construction of the $S$-matrix.
} 
The notation $H$ is reminiscent of the Higgs field, but one with no "vacuum condensate" (leaving aside the academic point of whether Higgs idea would allow for our terminology): $\langle 0|H(x)| 0>=0$, where $| 0>\in \mathcal{F}$ is the vacuum of the free fields. For the representation of $A_{a}(\overline{7}), u_{a}, \tilde{u}_{a}$ (33), $\phi_{a}$ (35) and $H$ (37) in a Krein Fock space $\mathcal{F}$ and especially the definition of $J$ we refer to [23 ${ }^{m}$. Note that the ghost fields $u_{a}, \tilde{u}_{a}$ (33) and $\phi_{a}$ (35) are also asymptotic fields, but that asymptotic states containing ghost exitations do not belong to the physical Hilbert space (14). From the commutation relations

$$
\begin{gathered}
{\left[Q, A_{a}^{\mu}\right]=i \partial^{\mu} u_{a}, \quad\left[Q, \phi_{a}\right]=i m_{a} u_{a}, \quad\left\{Q, u_{a}\right\}=0,} \\
\left\{Q, \tilde{u}_{a}\right\}=-i\left(\partial_{\mu} A_{a}^{\mu}+m_{a} \phi_{a}\right), \quad[Q, H]=0
\end{gathered}
$$

we conclude that $\mathcal{H}_{\text {phys }}$ (14) is the linear span of the set of states $B_{1} \ldots B_{l} \mid 0>$ ,$l \in \mathbf{N}_{0}$, where $B_{1}, \ldots, B_{l}$ are transversal vector meson fields (three polarizations) or $H$-fields. Using this explicit result the positivity assumption (13) can be verified [23]. We emphasize that $H$ is physical, in contrast to the (scalar) bosonic ghost fields $\phi_{a}$.

We are now looking for the possible interactions $W \equiv W_{0}$ which satisfy the following requirements:

(a) $W$ is a Wick polynomial in the free incoming fields. Each monomial in $W$ has at least three factors,

(b) $W$ is invariant with respect to Poincaré transformations,

(c) the number of $u$-fields agrees with the number of $\tilde{u}$-fields in each monomial of $W$ (i.e. the 'ghost number' is zero),

(d) the scaling degree (or mass dimension) of $W$ is $\leq 4$ (this is necessary for renormalizability by power counting),

(e) $W=W^{*}$ (which yields $S^{*}=S^{-1}$ if the time ordered products are suitably normalized),

(f) physical consistency (31).

First we point out that the requirements (a)-(f) do not fix the interaction uniquely. Even if we replace in (f) the physical consistency (31) by the $Q$ divergence condition (29-30), which is a stronger requirement, the following non-uniqueness is known: considering solely the $Q$-divergence condition to

\footnotetext{
${ }^{14}$ In [23] it was not realized that without the Higgs field $H$ (or an ingenious substitute) the $Q$-divergence condition to second order is violated. We represent the $H$-field similarly to the other scalar fields $\phi_{a}$ with $J=\mathbf{1}$ in the $H$-Fock space.
} 
first order (29), it obviously remains the freedom to add divergence and coboundary couplings to $W$

$$
W^{(\boldsymbol{\beta}, \boldsymbol{\gamma})}=W+\sum_{l} \beta_{l} \partial_{\nu} D_{l}^{\nu}+\sum_{j} \gamma_{j}\left\{Q, K_{j}\right\}, \quad \beta_{l}, \gamma_{j} \in \mathbf{R}
$$

where $D_{l}^{\nu}, K_{j}$ are restricted by (a)-(e). Taking additionally the $Q$-divergence condition to orders $n \geq 2$ (30) into account, it seems that this freedom can be maintained, due to the following result. It is shown in 15 that the Q-divergence condition (30) can be satisfied to all orders (by choosing suitable normalizations) for any $(\boldsymbol{\beta}, \boldsymbol{\gamma})$, if the 'generalized (free perturbative operator) gauge invariance' $⿴ 囗 十)$ holds true for $(\boldsymbol{\beta}, \boldsymbol{\gamma})=(\mathbf{0}, \mathbf{0})$. Moreover, under this assumption, one can prove

$P_{0} T\left(W^{(\boldsymbol{\beta}, \boldsymbol{\gamma})}\left(x_{1}\right) \ldots W^{(\boldsymbol{\beta}, \boldsymbol{\gamma})}\left(x_{n}\right)\right) P_{0}=P_{0} T\left(W^{(\mathbf{0}, \mathbf{0})}\left(x_{1}\right) \ldots W^{(\mathbf{0 , 0})}\left(x_{n}\right)\right) P_{0}+$ divergences

(see [15], 'divergences' means 'divergences of local operators') and hence the physical $S$-matrix $S_{00}$ is independent from $(\boldsymbol{\beta}, \boldsymbol{\gamma})$ (because the divergences vanish in the adiabatic limit in pure massive theories).

Now we make the most general Ansatz for $W$ (up to divergence and

\footnotetext{
${ }^{15}$ The 'generalized (free perturbative operator) gauge invariance' is the following statement. To the interaction density $W \equiv W_{0}$ there exist Wick polynomials $W_{1}^{\nu}$ and $W_{2}^{\mu \nu}$ with

$$
\left[Q, W_{0}\right]=i \partial_{\nu} W_{1}^{\nu}, \quad\left\{Q, W_{1}^{\nu}\right\}=i \partial_{\mu} W_{2}^{\mu \nu}, \quad\left[Q, W_{2}^{\mu \nu}\right]=0
$$

and the time ordered products of $W_{0}, W_{1}^{\nu}$ and $W_{2}^{\mu \nu}$ fulfil

$\left[Q, T\left(W_{j_{1}}\left(x_{1}\right) \ldots W_{j_{n}}\left(x_{n}\right)\right)\right]_{\mp}=i \sum_{l=1}^{n} \partial^{x_{l}} T\left(W_{j_{1}}\left(x_{1}\right) \ldots W_{j_{l}+1}\left(x_{l}\right) \ldots W_{j_{n}}\left(x_{n}\right)\right), \quad j_{1}, \ldots, j_{n} \in\{0,1,2\}$,

where we have the anticommutator on the l.h.s. iff $\left(j_{1}+\ldots+j_{n}\right)$ is odd, and where $T\left(\ldots W_{3}\left(x_{l}\right) \ldots\right) \equiv 0$ by definition. Similarly to (30), the second equation is a normalization condition on the time ordered products. A proof that it can be satisfied to all orders is still missing in any nonabelian model. Nevertheless we strongly presume that this statement holds true for all models which are BRST-invariant, especially for the model studied below of three massive, selfinteracting vector fields.
} 
coboundary terms ) which satisfies (a)-(e)

$$
\begin{aligned}
W & =f_{a b c}: A_{a \mu} A_{b \nu} \partial^{\nu} A_{c}^{\mu}:+f_{a b c}^{1}: u_{a} \partial^{\mu} \tilde{u}_{b} A_{c \mu}: \\
& +d_{a b c}\left(: A_{a}^{\mu} \phi_{b} \partial_{\mu} \phi_{c}:-: A_{a}^{\mu} \partial_{\mu} \phi_{b} \phi_{c}:\right)+e_{a b c}: A_{a}^{\mu} A_{b \mu} \phi_{c}: \\
& +h_{a b c}: \tilde{u}_{a} u_{b} \phi_{c}:+j_{a b c}: \phi_{a} \phi_{b} \phi_{c}:+k_{a b}\left(: H A_{a}^{\mu} \partial_{\mu} \phi_{b}:-: \partial_{\mu} H A_{a}^{\mu} \phi_{b}:\right) \\
& +l_{a b}: A_{a}^{\mu} A_{b \mu} H:+p_{a b}: H \tilde{u}_{a} u_{b}:+q_{a b}: H \phi_{a} \phi_{b}:+r_{a}: H^{2} \phi_{a}:+s: H^{3}: \\
& + \text { (quadrilinear terms, e.g. } \sim: A A A A:,: A A u \tilde{u}:,: A A \phi \phi:, H^{4}:\{41)
\end{aligned}
$$

where $f_{a b c}, f_{a b c}^{1}, d_{a b c}, e_{a b c}, h_{a b c}, j_{a b c}, k_{a b}, l_{a b}, p_{a b}, q_{a b}, r_{a}, s \in \mathbf{R}$ are arbitrary constants (which are introduced without any knowledge about a gauge group). One can show that physical consistency (31) requires that $f_{a b c}$ is totally antisymmetric (i.e. this part of the result (45) below is independent of the restriction to three vector fields which is made in the following). Hence the simplest non-trivial model of selfinteracting massive $\left(m_{a}>0 \forall a\right)$ vector fields is that of three fields: $a=1,2,3$. From now on we specialize to this case. In particular a single massive spin 1 field (corresponding to the U(1)-case) cannot be selfinteracting.

Our aim is to determine the parameters in $W$ (41) and the tree normalization terms (see (49) below) by the consistency condition (31). The hope is that one can conclude from

$$
0=\left.\left[Q, S_{n}\right]\right|_{\operatorname{ker} Q}=\left.\frac{i^{n}}{n !} \int d x_{1} \ldots d x_{n}\left[Q, T\left(W\left(x_{1}\right) \ldots W\left(x_{n}\right)\right)\right]\right|_{\operatorname{ker} Q}
$$

that $\left[Q, T\left(W\left(x_{1}\right) \ldots W\left(x_{n}\right)\right)\right]$ must be a sum of divergences of local operators and that then one can follow the calculations in [2].

But there is a difficulty at first order, which is explained in detail in appendix A. The adiabatic limit of the trilinear terms in $W$ vanishes (except possibly the $H$-couplings) due to energy-momentum conservation. This is just the fact that for stable particles there are no S-matrix elements with three particle legs; the lowest tree contributions involve $\geq 4$ legs. Hence, we get no information about these terms from $\left.\left[Q, S_{1}\right]\right|_{\operatorname{ker} Q}=0$. For the quadrilinear terms $W^{(4)}$ in $W$ the procedure works (see also appendix A): we obtain that $\left[Q, W^{(4)}\right]$ must be the divergence of a Wick polynomial. In [20] it is shown that this implies $W^{(4)}=0$ except for a term $\sim: H^{4}:$ But the

\footnotetext{
${ }^{16}$ The resulting model is usually called ' $S U(2)$ Higgs-Kibble model'. It is obtained from the electroweak theory, which is studied in detail in [2], by setting the Weinberg angle $\Theta_{W}=0$ and omitting the photon field, which decouples in this case.
} 
latter term will be excluded by consistency (31) to second order. (The wellknown : $H^{4}$ :-coupling is of second order in $g$, it appears in the framework of causal perturbation theory as a tree normalization term of $T\left(W\left(x_{1}\right) W\left(x_{2}\right)\right)$, see below.)

To determine the parameters in the trilinear terms $W^{(3)}$ of $W$ (41) we compute the tree diagrams in $T\left(W^{(3)}\left(x_{1}\right) W^{(3)}\left(x_{2}\right)\right.$ ) (which depend on these parameters) and require

$$
\left.\int d^{4} x_{1} d^{4} x_{2}\left[Q,\left.T\left(W^{(3)}\left(x_{1}\right) W^{(3)}\left(x_{2}\right)\right)\right|_{\text {tree }}\right]\right|_{\operatorname{ker} Q}=0 .
$$

Instead of the long explicit calculation we give a heuristic description:

- For the terms with $x_{1} \neq x_{2}$ the $T$-product factorizes, e.g. for $x_{1} \notin$ $x_{2}+\bar{V}^{-}$we have

$$
\left.\left[Q,\left.T\left(W^{(3)}\left(x_{1}\right) W^{(3)}\left(x_{2}\right)\right)\right|_{\text {tree }}\right]=\left.\left[Q, W^{(3)}\left(x_{1}\right)\right] W^{(3)}\left(x_{2}\right)\right|_{\text {tree }}+W^{(3)}\left(x_{1}\right)\left[Q, W^{(3)}\left(x_{2}\right)\right)\right]\left.\right|_{\text {tree }} \text {. }
$$

This makes it plausible that the cancelation of these terms $\square$ yields the same restrictions as the $Q$-divergence condition to first order $(29):\left[Q, W^{(3)}\right]=$ $i \partial_{\nu} W_{1}^{(3) \nu}$ for some Wick monomial $W_{1}^{(3) \nu}$. 1 In this way we obtain

$$
\begin{aligned}
& f_{a b c}=-f_{a b c}^{1} \text { and } f_{a b c} \quad \text { is totally antisymmetric, } \\
& d_{a b c}=f_{a b c} \frac{m_{b}^{2}+m_{c}^{2}-m_{a}^{2}}{4 m_{b} m_{c}}, \quad e_{a b c}=f_{a b c} \frac{m_{b}^{2}-m_{a}^{2}}{2 m_{c}} \\
& h_{a b c}=f_{a b c} \frac{m_{a}^{2}+m_{c}^{2}-m_{b}^{2}}{2 m_{c}}, \quad j_{a b c}=0, \quad r_{a}=0
\end{aligned}
$$

and some relations for $k_{a b}, l_{a b}, p_{a b}$ and $q_{a b}$ ([2], [20] and [30]). There results no restriction on $s$. One easily verifies that these values of the parameters are not only necessary for the cancelation of the terms $x_{1} \neq x_{2}$ in (43) and for (29), they are also sufficient. By absorbing a constant factor in $g$ we obtain

$$
f_{a b c}=\epsilon_{a b c} .
$$

\footnotetext{
${ }^{17}$ The fact that the terms $x_{1} \neq x_{2}$ cannot be canceled by diagonal terms $x_{1}=x_{2}$ becomes clear from the explicit expressions.

${ }^{18} \mathrm{It}$ is obvious that the $Q$-divergence condition to first order implies the cancelation of the terms $x_{1} \neq x_{2}$ in $(43)$, but here we proceed in the opposite direction.
} 
The latter are the structure constants of $s u(2)$. So the gauge group structure is not put in, it comes out as a consequence of physical consistency and the Ansatz (41) for $W$. For more complicated models (i.e. more than three vector fields) this conclusion is impossible at this stage, because one does not know that the $f_{a b c}$ 's satisfy the Jacobi identity. But then the latter is obtained in the next step (see below). So far it is possible that all couplings involving the $H$-field vanish, i.e. the Higgs field is not yet needed.

- The remaining terms in (43) come from the diagonal $x_{1}=x_{2}$. Their cancelation cannot be achieved without $H$-couplings. This condition yields important results: ${ }^{\text {To }}$

- The $f_{a b c}$ 's must fulfil the Jacobi identity. (In our simple model this is already known (46).)

- The masses must agree

$$
m \equiv m_{1}=m_{2}=m_{3}
$$

- The $H$-coupling parameters take the values

$$
k_{a b}=\frac{\kappa}{2} \delta_{a b}, \quad l_{a b}=-\frac{\kappa m}{2} \delta_{a b}, \quad p_{a b}=\frac{\kappa m}{2} \delta_{a b}, \quad q_{a b}=\frac{\kappa m_{H}^{2}}{4 m} \delta_{a b}
$$

where $\kappa \in\{-1,1\}$. The parameter $s$ is still free.

- There is no term $\sim: H^{4}:$ in $W$ (i.e. in first order in $g$ ).

- In $\left.T\left(W\left(x_{1}\right) W\left(x_{2}\right)\right)\right|_{\text {tree }}$ the C-number distributions are Feynman propagators with derivatives: $\Delta^{F}\left(x_{1}-x_{2}\right), \partial_{\mu} \Delta^{F}\left(x_{1}-x_{2}\right)$ and $\partial_{\nu} \partial_{\mu} \Delta^{F}\left(x_{1}-x_{2}\right)$. The first two extend uniquely to the diagonal $x_{1}=x_{2}$ and the last one has a distinguished extension, namely $\partial_{\nu} \partial_{\mu} \Delta^{F}\left(x_{1}-x_{2}\right)$ [2. We denote this extension by $\left.T\left(W\left(x_{1}\right) W\left(x_{2}\right)\right)\right|_{\text {tree }} ^{0}$. So-called 'tree normalization terms'

$$
N\left(x_{1}, x_{2}\right)=C \delta\left(x_{1}-x_{2}\right): B_{1}\left(x_{1}\right) B_{2}\left(x_{2}\right) B_{3}\left(x_{3}\right) B_{4}\left(x_{4}\right):, \quad C \in \mathbf{R} \text { or } i \mathbf{R}
$$

\footnotetext{
${ }^{19}$ These results agree precisely with the ones derived from the $Q$-divergence condition (for second order tree diagrams) in [2], [30] and with [20].

${ }^{20}$ Stora [41] found (for an arbitrary number of massless selfinteracting vector fields) that the $Q$-divergence condition to first order implies that the coupling parameters are totally antisymmetric and that the $Q$-divergence condition for second order tree diagrams yields the Jacobi identity.

${ }^{21}$ The general extension which is Poincaré covariant (D) and does not increase the scaling degree (F) reads: $\partial_{\nu} \partial_{\mu} \Delta^{F}\left(x_{1}-x_{2}\right)+C g_{\nu \mu} \delta\left(x_{1}-x_{2}\right), \quad C \in \mathbf{C}$ arbitrary.
} 
$\left(B_{1}, \ldots, B_{4} \in\left\{A^{\mu}, u, \tilde{u}, \phi, H\right\}\right)$ can be added to $\left.T\left(W\left(x_{1}\right) W\left(x_{2}\right)\right)\right|_{\text {tree }} ^{0}$, if they satisfy the properties (b) (Poincaré covariance), (c) (ghost number), (d) (scaling degree) and (e) (unitarity) which are required above for $W$ (here they restrict $N\left(x_{1}, x_{2}\right)$ ). These tree normalization terms (49) correspond to the quadrilinear terms of order $g^{2}$ ( $g$ denotes the coupling constant) in the interaction Lagrangian of the conventional theory, they have the same influence on the perturbation series of the $S$-matrix. The cancelation of the terms $x_{1}=x_{2}$ in (43) fixes the possible tree normalization terms (i.e. the constants $C$ in (49)) uniquely (in terms of $s$ ), except for 2

$$
N_{H^{4}}\left(x_{1}, x_{2}\right)=\lambda \delta\left(x_{1}-x_{2}\right): H^{4}\left(x_{1}\right):, \quad \lambda \in \mathbf{R} .
$$

- The parameters $s$ and $\lambda$, which are still free, are determined by physical consistency (42) for the tree diagrams to third order (analogously to [2]):

$$
s=\frac{m_{H}^{2}}{4 m}, \quad \lambda=-\frac{m_{H}^{2}}{16 m^{2}} .
$$

So $W$ and the tree diagram normalizations to second order are completely determined (up to the sign $\kappa$, which is conventional) and these terms agree precisely with the interaction Lagrangian obtained by the Higgs mechanism. We have made the assumption that there is at most one physical scalar field $H$. This assumption is most probably not necessary. By specializing the results of [30] to our model (of three selfinteracting massive vector fields) one finds that there is no solution of the $Q$-divergence condition with more than one $H$-field, provided the $H$-fields couple to the vector or ghost fields.

The Higgs potential is not put in here, it is derived from physical consistency. Spontaneous symmetry breaking plays no role in this approach, because we start with the massive free incoming fields. A proof that physical consistency can be satisfied to all orders (by choosing suitable normalizations) is missing up to now, but we are convinced that this holds true.

\section{Renormalizability and Ghosts}

The formulation of massive selfinteracting vector mesons of the previous section will now serve as a point of departure for a more fundamental conceptual

\footnotetext{
${ }^{22}$ For the expert we mention that tree normalization terms with $B_{1}, \ldots, B_{4}$ exclusively scalar fields (e.g. $N_{H^{4}}$ ) are required. In contrast to the other tree normalization terms (i.e. the tree normalization terms with vector field factors) they violate the normalization condition (N3) in [10] (or (43) in 17]). But this is no harm.
} 
discussion. Similar to Weinberg (Weinberg's old work on Feynman rules for higher spin) we start with Wigner's theory of particle representations; in our case because we want to avoid any parallelism to (quasi)classical systems (quantization) and (for reasons which will become gradually clear to the reader) develop local quantum physics from an intrinsic point of view as much as possible. In particular we would like to understand the curious phenomenon that, contrary to the classical situation, the possibilities of perturbative renormalizable QFT are the more restrictive, the higher the spin. Whereas the classical theory is in need of an additional selection principle (the gauge principle in case of zero mass), local quantum physics for spin $\geq 1$ is more restrictive: the particle content and renormalizability fix the vector meson theory (where the possible triviality for spin $>1$ is a special case It is this result red backward into (quasi)classical field theory, which, in the spirit of Bohr's correspondence principle gives a fundamental physical support for the classical gauge selection principle (which then gives the strong link with the mathematical-aesthetical appeal to fibre bundles). As a side result we will learn that the massive theory fulfills the Schwinger-Swieca [38] 433 screening mechanism, and that (similar to the gauge interpretation in terms of a Higgs field) the theory has more physical degrees of freedom than the massive vector mesons from which we started in zeroth order, namely consistent perturbation theory requires the introduction of a scalar $H$-field (the Higgs field without Higgs condensate ${ }^{24}$ ) of the previous section.

It is well-known that the step from the Wigner representation theory of particles (positive energy irreducible representations of the Poincaré group with finite spin/helicity) to local free fields is described in terms of intertwiners $u, v^{20}$

$$
\psi^{\left[n_{+}, n_{-}\right]}(x)=\int \sum_{s_{3}=-s}^{s}\left\{e^{-i p x} u\left(\vec{p}, s_{3}\right) a\left(\vec{p}, s_{3}\right)+e^{i p x} v\left(\vec{p}, s_{3}\right) b^{*}\left(\vec{p}, s_{3}\right)\right\} \frac{d^{3} p}{2 \omega}
$$

which intertwine the Wigner representation matrices $D^{(s)}(R(\Lambda, p))$ with ma-

\footnotetext{
${ }^{23}$ In a recent paper Scharf and Wellmann [31] have shown that there exists no renormalizable theory for $\mathrm{s}=2$ which satisfies the free perturbative operator gauge invariance.

${ }^{24}$ We use the notation $H$-field only in order to avoid any association with Higgs condensates.

${ }^{25}$ The letter $u$, which was used in the previous section for a ghost field, means here an intertwiner. The ghost fields in the Wigner one-particle space will be denoted by $(\omega, \bar{\omega}, \varphi)$. The corresponding Fock space fields are the fields $(u, \tilde{u}, \phi)$ of the previous section.
} 
trices of the covariant Lorentz group representation $D^{\left[n_{+}, n_{-}\right]}(\Lambda)$

$$
D^{\left[n_{+}, n_{-}\right]}(\Lambda) u(p)=u(\Lambda p) D^{(s)}(R(\Lambda, \Lambda p))
$$

where for convenience we have collected the $(2 s+1) u^{\left(n_{+}, n_{-}\right)}(p)$ mixed (un)dotted $\left(2 n_{+}+1\right)\left(2 n_{-}+1\right)$ component $\mathrm{u}$-spinors into a rectangular $\left(2 n_{+}+1\right)\left(2 n_{-}+\right.$ $1) \times(2 s+1)$ matrix $u(p)$ and similar for the $v^{\prime} s$.

Let us first note that the covariantized inner product in the one-particle Wigner space for $s \geq 1$ contains necessarily first or higher powers of momenta. Associated with this is the fact that the intertwiners $u, v$ have a dimension $\geq 1$ which immediately translates into an operator dimension of the field $\operatorname{dim} \psi \geq 2$. Since interaction densities $W_{0} \equiv W$ are at least trilinear in free fields and since the smallest possible operator dimension is 1 (for scalar fields), it is impossible to satisfy the renormalizability condition $\operatorname{dim} W \leq 4$ within the Stückelberg-Bogoliubov-Epstein-Glaser operator approach. Inspired by the idea of a cohomological representation of the physical space and the physical observables in the previous section one looks for a cohomological extension of the Wigner space for massive vector mesons in order that the associated two-point function (or propagation kernel) has a milder (renormalizable) high momentum behavior. The results of the previous section also suggest the simplest possibility to achieve that, namely to use three additional indefinite metric scalar wave functions (where on the Wigner level the "statistics" is yet undetermined). More precisely we form an extended Hilbert space $H_{\text {ext }}$ by the multicomponent wave functions $\left(A_{\mu}, \omega, \bar{\omega}, \varphi\right)$ defined on the mass shell. $H_{\text {ext }}$ has in addition to a positive definite inner product another one which does not have this property and corresponds to the Krein structure of the previous section. The latter is relevant in order to have at least some pseudo-unitary Lorentz covariance and a definition of modular localized subspaces. On $H_{\text {ext }}$ we define a BRS-like operator by

$$
s_{W}\left(\begin{array}{l}
A_{\mu}^{a}(p) \\
\omega_{a}(p) \\
\bar{\omega}_{a}(p) \\
\varphi_{a}(p)
\end{array}\right)=\left(\begin{array}{l}
p_{\mu} \omega_{a}(p) \\
0 \\
-p^{\mu} A_{\mu}^{a}(p)-i m_{a} \varphi_{a}(p) \\
i m_{a} \omega_{a}(p)
\end{array}\right) .
$$

The so defined $s_{W}$-operation defines a differential space since the definition easily leads to $s_{W}^{2}=0$. One then uses this $s_{W}$ in order to write the following cohomological representation for the physical (Wigner) Hilbert space $H_{W}$ in 
terms of the above extended space $H_{\text {ext }}$

$$
\begin{aligned}
H_{W} & =\frac{\operatorname{ker} s_{W}}{\operatorname{ran} s_{W}} \\
& =c l \cdot\left\{A_{\mu}(p) \mid p^{\mu} A_{\mu}(p)=0,-\int A_{\mu}(p) A^{\mu}(p) \frac{d^{3} p}{2 \omega}<\infty\right\} \\
& =c l .\left\{A_{\mu}(p) \mid-\int A_{\mu}(p)\left(g^{\mu \nu}-\frac{p^{\mu} p^{\nu}}{m^{2}}\right) A_{\nu}(p) \frac{d^{3} p}{2 \omega}<\infty\right\}
\end{aligned}
$$

i.e. we obtain the $L^{2}$-closure of the space of transversal vector wave functions which in terms of the associated fields (52) had the high $\operatorname{dimension} \operatorname{dim} A=2$ which was responsible for the lack of renormalizable interactions within the original (non-extended) formulation. (The transversality condition does not lower the dimension of the vector wave functions or the corresponding fields.) On the other hand the extended Hilbert space has no transversality condition and obeys the classical assignment of dimensions (i.e. $\operatorname{dim} A=1$ in $H_{e x t}$ ). This is due to the fact that ghost contributions damp the high momentum behavior of the associated two-point function. ${ }^{20}$

The extension has however an influence on the modular localization theory in Wigner space where the latter is (via the CCR/CAR functor) the preempted locality in Fock space. For example the wedge (Rindler or BisognanoWichmann) localized subspace involves instead of the simple complex-conjugation in addition the Krein operator $\eta$ i.e. one has for the action of the pre-Tomita operator ${ }^{27}$

$$
(S \psi)(p)=(\eta \bar{\psi})(p)
$$

\footnotetext{
${ }^{26}$ In the Lagrangean framework there is an alternative method to lower the dimension of the massive vector field $A$ from 2 to 1 . In contrast to massless vector fields, the Proca field $\left(\mathcal{L}=-\frac{1}{4} F^{2}+\frac{1}{2} m^{2} A^{2}, F^{\mu \nu} \equiv \partial^{\mu} A^{\nu}-\partial^{\nu} A^{\mu}\right)$ has a well-defined propagator without introducing a "gauge fixing term", but it has $\operatorname{dimension} \operatorname{dim} A=2$. Stückelbergs trick is to add a "gauge fixing term" $\mathcal{L}_{1}=-\frac{1}{2} \lambda(\partial \cdot A)^{2}, \lambda>0$ (this misleading terminology is chosen because because $\mathcal{L}_{1}$ has the same form as the gauge fixing Lagrangean in the massless case), which damps the high momentum behavior of the propagator such that $\operatorname{dim} A=1$. In this Stückelberg formalism, the necessity to introduce ghosts shows up in the fact that without ghosts it is impossible to define a stable physical subspace or factor space. For $\lambda=1$ (Feynman gauge) (7) one obtains the same free vector fields as in the quantization of the cohomological extension of the Wigner one-particle space. We prefer the latter method, because we do not want to rely on a Lagrangean framework, instead we want to be close to the particle picture.

${ }^{27}$ The pre-Tomita operator $S$ has nothing to do with the above nilpotent operators $s_{W}$ (54) (in the one particle Wigner space) or $s$ (57) in Fock space.
} 
Where $\psi$ is the multi-component wave function involving the ghosts in addition to the vector potential. The standard modular localization theory can be found in [33] and the adaptation to the present extended "pseudo-modular" case will be treated in a separate paper. The physical Wigner subspace (more precisely it is a cohomologically defined factor space) is precisely characterized by the validity of the "correct" modular localization associated with the Tomita theory. Although our ghost extension of the $(\mathrm{m}, \mathrm{s}=1)$ Wigner representation is not uniquely fixed (we chose a "minimal" extension) we believe that any other cohomological extension which also lowers the $\operatorname{dim} A=2$ down to its classical value $\operatorname{dim} A=1$ will contain the minimal and possible additional pieces which do not change the physical content ${ }^{28}$.

The next step from particles to fields is the answer to the question of what is the action of $s_{W}$ on the multi particle tensor space [9]. From the usual Fock space formalism we are used to the following action of derivations $\delta$ on tensor products

$$
\delta\left(\psi_{1} \otimes \psi_{2}\right)=\delta \psi_{1} \otimes \psi_{2}+\psi_{1} \otimes \delta \psi_{2}
$$

It is easy to see that the tensor product action of $s_{W}$ must include a grading in order to maintain the nilpotency

$$
s\left(\psi_{1} \otimes \psi_{2}\right)=s \psi_{1} \otimes \psi_{2}+\psi_{1}(-)^{\text {degree } \psi_{1}} \otimes s \psi_{2},
$$

where $s$ denotes the Fock space version of $s_{W}$.2 So, two of the three scalar ghost fields $(u, \tilde{u}, \phi)^{30}$, which are companions of each massive vector meson field, are required to be graded fermionic fields and the third one must be bosonic.

In this way we obtain the Fock space formalism of the previous section with $[Q, \cdot]$ being the implementation of $s$ in Fock space. Whereas the ghost formalism can be pursued back into the Wigner one-particle theory, the necessity to choose trilinear couplings at first order in $g$ with coefficients fulfilling group theoretical symmetry, as well as the necessity of enlargement of the

\footnotetext{
${ }^{28}$ The geometrical Faddeev-Popov method can also be considered as a minimal extension of the functional measure. It owes its unique appearance more to geometric than quantum physical reasoning.

${ }^{29}$ ¿From the context it should be clear whether we mean by the letter $s$ the present nilpotent Fock space operator $s$ or the spin.

${ }^{30} \mathrm{We}$ recall that $(u, \tilde{u}, \phi)$ are the Fock space fields of the previous section and correspond to $(\omega, \bar{\omega}, \varphi)$ in the Wigner one-particle space.
} 
vector meson setup by additional physical degrees of freedom (whose simplest and perhaps only realization are the scalar $H$-fields) only shows up as a consistency requirement above the zeroth order.

\section{Determination by Field Content}

In the second section physical consistency was formulated for the $S$-matrix (31). We are now looking for a corresponding condition in terms of interacting fields. Such fields (including composites) are defined by means of the Bogoliubov transition functional $S(\mathbf{g})$ (1) in Fock space as formal power series in $g_{0}$. The interacting field $W_{j \text { int }}\left(x ; g_{0} W\right)$ due to the interaction $W \equiv W_{0}$ and corresponding to the Wick polynomial $W_{j}, j=1, \ldots, G$ of free fields, is defined by

$$
\left.W_{j \text { int }}\left(x ; g_{0} W\right) \equiv \frac{\delta}{i \delta g_{j}(x)} S\left(g_{0}, 0, \ldots, 0\right)^{-1} S\left(g_{0}, 0, \ldots, g_{j}, 0, \ldots\right)\right|_{g_{j}=0} .
$$

By inserting (1) one obtains the perturbative expansion of the interacting fields

$W_{j \text { int }}\left(x ; g_{0} W\right)=W_{j}(x)+\sum_{n=1}^{\infty} \frac{i^{n}}{n !} \int d^{4} x_{1} \ldots d^{4} x_{n} g_{0}\left(x_{1}\right) \ldots g_{0}\left(x_{n}\right) R\left(W\left(x_{1}\right) \ldots W\left(x_{n}\right) ; W_{j}(x)\right)$,

with the 'totally retarded products'

$R\left(A_{1}\left(x_{1}\right) \ldots A_{n}\left(x_{n}\right) ; A(x)\right) \equiv \sum_{I \subset\{1, \ldots, n\}}(-1)^{|I|} \bar{T}\left(A_{i}\left(x_{i}\right), i \in I\right) T\left(A_{k}\left(x_{k}\right), k \in I^{c}, A(x)\right)$

where $A_{1}, \ldots, A_{n}, A$ are Wick polynomials, $I^{c} \equiv\{1, \ldots, n\} \backslash I$ and $\bar{T}$ denotes the 'anti-chronological product'. The corresponding generating functional is $S(\mathbf{g})^{-1}$. The anti-chronological products can be obtained uniquely from the time ordered products by the usual inversion of a formal power series

$$
\bar{T}\left(A_{1}\left(x_{1}\right) \ldots A_{n}\left(x_{n}\right)\right)=\sum_{P \in \operatorname{Part}\{1, \ldots, n\}}(-1)^{|P|+n} \prod_{p \in P} T\left(A_{i}\left(x_{i}\right), i \in p\right) .
$$


By means of causality (4) one easily finds that the $R$-products (60) have totally retarded support with respect to the distinguished coordinate $\mathrm{x}$.

$$
\operatorname{supp} R\left(A_{1}\left(x_{1}\right) \ldots A_{n}\left(x_{n}\right) ; A(x)\right) \subset\left\{\left(x_{1}, \ldots x_{n} ; x\right) \mid x_{i} \in x+\bar{V}_{-}, \forall i=1, \ldots n\right\} .
$$

In a pure massive theory the strong adiabatic limit of the interacting fields exists as a formal power series in $g:=g_{0}(0)$ :

$W_{j \text { int }}(x) \psi \equiv W_{j \text { int }}(x ; W) \psi \equiv \lim _{\epsilon \rightarrow 0} W_{j \text { int }}\left(x ; g_{0 \epsilon} W\right) \psi, \quad$ where $\quad g_{0 \epsilon}(x) \equiv g_{0}(\epsilon x), \psi \in \mathcal{D}$,

because this holds true for $S(\mathbf{g})$ (6) 18.

A *-symmetrical interacting field in the adiabatic limit (i.e. $\phi_{\text {int }}(x) \equiv$ $\left.\phi_{\text {int }}(x ; W)=\lim _{\epsilon \rightarrow 0} \phi_{\text {int }}\left(x ; g_{0 \epsilon} W\right), \phi_{\text {int }}^{*}=\phi_{\text {int }}\right)$ is called physical (or an observable) if it induces a well-defined operator on the factor space $\mathcal{H}_{\text {phys }}^{\prime}(22)$. This holds true iff

$$
\phi_{\text {int }}(f ; W) \operatorname{ker} Q \subset \operatorname{ker} Q \wedge \phi_{\text {int }}(f ; W) \operatorname{ran} Q \subset \operatorname{ran} Q, \quad \forall f \in \mathcal{S}\left(\mathbf{R}^{4}\right)
$$

(cf.(25)). This is equivalent to

$$
\left.\left[Q, \phi_{\text {int }}(f ; W)\right]\right|_{\text {ker } Q}=0, \quad \forall f \in \mathcal{S}\left(\mathbf{R}^{4}\right) .
$$

(The nontrivial part of this statement is that (65) implies $\phi_{\text {int }}(f) \operatorname{ran} Q \subset$ $\operatorname{ran} Q$. However, using the notations of sect. 2, the condition (65) is equivalent to $\phi_{\text {int }}(f)_{+-}=0=\phi_{\text {int }}(f)_{+0}$. Hence $\phi_{\text {int }}(\bar{f})_{0-}=\left(\phi_{\text {int }}(f)_{+0}\right)^{*}=0$. Together we obtain $\phi_{\text {int }}(f) \operatorname{ran} Q \subset \operatorname{ran} Q$.)

Let $F_{a}^{\mu \nu} \equiv \partial^{\mu} A_{a}^{\nu}-\partial^{\nu} A_{a}^{\mu}$. We now require that (for each $\left.a=1, \ldots, M\right)$ there exists a physical field $\mathcal{F}_{a \text { int }}^{\mu \nu}$, i.e.

$$
\left.\left[Q, \mathcal{F}_{a \text { int }}^{\mu \nu}(x ; W)\right]\right|_{\operatorname{ker} Q}=0,
$$

with the additional properties:

(i) $\mathcal{F}_{a \text { int }}^{\mu \nu}=F_{a \text { int }}^{\mu \nu}+\sum_{k} c_{k} \psi_{a k \text { int }}^{\mu \nu}$, where the $c_{k}$ are formal power series of (constant) complex numbers and $\mathcal{F}_{a \text { int }}^{\mu \nu}$ agrees in zeroth order with the free $F_{a}^{\mu \nu}$, i.e. the $c_{k}$ vanish to zeroth order: $c_{k}^{(0)}=0$; 
(ii) the zeroth order $\psi_{a k}^{\mu \nu}$ of $\psi_{a k \text { int }}^{\mu \nu}$ is a Wick monomial which has precisely one factor $F$ or $A$, the other factors are ghost or scalar fields, and $\psi_{a k}^{\mu \nu} \neq$ $F_{b}^{\mu \nu}, \forall a, b \in\{1, \ldots, M\}$;

(iii) the scaling degree (or mass dimension) of $\psi_{a k}$ is $\leq 4$;

(iv) $\mathcal{F}_{a \text { int }}^{\mu \nu}$ is a Lorentz tensor of second rank and is anti-symmetrical in $(\mu, \nu)$;

(v) the ghost number of $\mathcal{F}_{a \text { int }}^{\mu \nu}$ is zero;

(vi) $\mathcal{F}_{a \text { int }}^{\mu \nu}(x ; W)^{*}=\mathcal{F}_{a \text { int }}^{\mu \nu}(x ; W)$.

The requirement (ii) is badly motivated (except the demand that $\psi_{a k}$ must be a Wick monomial), its main purpose is to shorten the calculations. However, the condition $\psi_{k}^{\mu \nu} \neq F^{\mu \nu}$ in (ii) is necessary for the uniqueness of $\mathcal{F}_{\text {int }}$ : the fields

$$
\left(1+\sum_{k=1}^{\infty} b_{k} g^{k}\right) \mathcal{F}_{a \text { int }}^{\mu \nu}, \quad b_{k}=\text { const. } \in \mathbf{R}
$$

satisfy all other requirements if $\mathcal{F}_{\text {int }}$ does so. It is an interesting question how far (ii) can be weakened such that the uniqueness of $\mathcal{F}_{\text {int }}$ does not get lost.

Due to the normalization condition (F) (scaling degree) for the time ordered products, the property (iii) implies $\operatorname{dim} \mathcal{F}_{a \text { int }}^{\mu \nu} \leq 4$. To specify (iv), (v) and (vi) note that they must be fulfilled in particular by the Wick monomials $\psi_{a k}$ (in the case of (vi) the coefficients $c_{k}$ are also involved: $c_{k}^{*} \psi_{a k}^{*}=c_{k} \psi_{a k}$ ). However, these three requirements also restrict the normalization of the higher orders of $\psi_{a k \text { int }}^{\mu \nu}$ and $F_{a \text { int }}^{\mu \nu}$. It is easy to see that these additional normalization conditions can be fulfilled (e.g. by antisymmetrization in $(\mu, \nu)$ of an arbitrary Poincaré covariant extension) and we assume that the normalizations are always done in such a way.

The most general Ansatz which is compatible with (i)-(vi) reads

$$
\begin{aligned}
\mathcal{F}_{d \mathrm{int}}^{\mu \nu}= & F_{d \mathrm{int}}^{\mu \nu}+t_{d a b}\left(F_{a}^{\mu \nu} \phi_{b}\right)_{\mathrm{int}}+\tilde{t}_{d a b}\left(A_{a}^{\mu} \partial^{\nu} \phi_{b}-A_{a}^{\nu} \partial^{\mu} \phi_{b}\right)_{\mathrm{int}} \\
+v_{d a b c}(: & \left.F_{a}^{\mu \nu} \phi_{b} \phi_{c}:\right)_{\mathrm{int}}+\tilde{v}_{d a b c}\left(: A_{a}^{\mu} \partial^{\nu} \phi_{b} \phi_{c}:-: A_{a}^{\nu} \partial^{\mu} \phi_{b} \phi_{c}:\right)_{\mathrm{int}} \\
+w_{d a b c}(: & \left.F_{a}^{\mu \nu} u_{b} \tilde{u}_{c}:\right)_{\mathrm{int}}+\tilde{w}_{d a b c}\left(: A_{a}^{\mu} u_{b} \partial^{\nu} \tilde{u}_{c}:-: A_{a}^{\nu} u_{b} \partial^{\mu} \tilde{u}_{c}:\right)_{\mathrm{int}} \\
+w_{d a b c}^{\prime}(: & \left.A_{a}^{\mu} \partial^{\nu} u_{b} \tilde{u}_{c}:-: A_{a}^{\nu} \partial^{\mu} u_{b} \tilde{u}_{c}:\right)_{\mathrm{int}} \\
& +x_{d a}\left(F_{a}^{\mu \nu} H\right)_{\mathrm{int}}+\tilde{x}_{d a}\left(A_{a}^{\mu} \partial^{\nu} H-A_{a}^{\nu} \partial^{\mu} H\right)_{\mathrm{int}} \\
& +y_{d a b}\left(F_{a}^{\mu \nu} \phi_{b} H\right)_{\mathrm{int}}+\tilde{y}_{d a b}\left(A_{a}^{\mu} \partial^{\nu} \phi_{b} H-A_{a}^{\nu} \partial^{\mu} \phi_{b} H\right)_{\mathrm{int}} \\
& +y_{d a b}^{\prime}\left(A_{a}^{\mu} \phi_{b} \partial^{\nu} H-A_{a}^{\nu} \phi_{b} \partial^{\mu} H\right)_{\mathrm{int}} \\
+z_{d a}(: \quad & \left.F_{a}^{\mu \nu} H^{2}:\right)_{\mathrm{int}}+\tilde{z}_{d a}\left(: A_{a}^{\mu} \partial^{\nu} H H:-: A_{a}^{\nu} \partial^{\mu} H H:\right)_{\mathrm{int}},
\end{aligned}
$$


where $t_{d a b}, \ldots, w_{d a b c}^{\prime}, x_{d a}, \ldots, \tilde{z}_{d a}$ are (arbitrary) constants which are formal power series in $\mathbf{R}$

$$
t_{d a b}=\sum_{k=1}^{\infty} t_{d a b}^{(k)} g^{k}, \quad x_{d a}=\sum_{k=1}^{\infty} x_{d a}^{(k)} g^{k}, \quad \text { etc.. }
$$

We define

$$
\mathcal{F}_{d}^{\mu \nu(k)} \equiv t_{d a b}^{(k)} F_{a}^{\mu \nu} \phi_{b}+\ldots+x_{d a}^{(k)} F_{a}^{\mu \nu} H+\ldots \quad \forall k \geq 1,
$$

hence ${ }^{31}$

$$
\mathcal{F}_{d \text { int }}^{\mu \nu}=F_{d \text { int }}^{\mu \nu}+\sum_{k=1}^{\infty} \mathcal{F}_{d \text { int }}^{\mu \nu(k)} g^{k}
$$

By definition we may assume $v_{d a b c}=v_{d a c b}$.

We require that the interaction density $W$ satisfies the properties (a)-(e) listed in section 2. The physical consistency of the $S$-matrix $\left.[Q, S]\right|_{\operatorname{ker} Q}=0$ is omitted here, we replace it by $\left[Q, \mathcal{F}_{\text {int }}(x ; W)\right]=0$ (see (69) below). In particular we require $\operatorname{dim} W \leq 4$ which ensures renormalizability. Additionally we assume that $W$ contains no quadrilinear terms. Most probably this assumption is not necessary, i.e. the other requirements (including the physicality (66)) exclude the quadrilinear terms. But this still needs to be checked and will be left open here.

We now replace the requirement (66) by

$$
\left[Q, \mathcal{F}_{a \text { int }}^{\mu \nu}(x ; W)\right]=0 .
$$

This may be justified in the following way 52 . Since we are working in the adiabatic limit our $Q$ (36) (constructed in terms of incoming free fields) agrees with the Kugo-Ojima operator $Q_{\text {int }}$ 25], 10 which implements the BRSTtransformation of the interacting fields. Hence $\left[Q, \mathcal{F}_{a \text { int }}^{\mu \nu}(x ; W)\right]$ is again a local operator. But by the Reeh-Schlieder theorem [27 such an operator is zero if it vanishes on the vacuum, which is an element of the kernel of $Q$. Thus the requirements (66) and (69) are equivalent. 53

\footnotetext{
${ }^{31}$ By definition the map $\phi \rightarrow \phi_{\text {int }}\left(\phi\right.$ a Wick monomial) is linear with $C^{\infty}$-functions as coefficients (here the coefficients are constants).

${ }^{32}$ We thank Klaus Fredenhagen for bringing this argument to our attention.

${ }^{33}$ This argument cannot be applied to $\left.[Q, S]\right|_{\text {ker } Q}=0$ (31), because the $S$-matrix is non-local.
} 
The main (completely new) result of this section is that the physicality (69) fixes the parameters $t_{d a b}, \ldots, \tilde{z}_{d a}$ in the Ansatz (67) and the interaction $W$ (including the tree normalization terms to second order (49)) up to the same non-uniqueness as in section 2, namely the addition of divergence- and coboundary couplings to $W(\sqrt[39]{)}$. Especially we will see that an additional physical degree of freedom is required: the Higgs field $H$ is needed in the interaction $W$ as well as in the field $\mathcal{F}_{d \text { int }}^{\mu \nu}$.

We verify this statement in appendix B by explicit calculation of the tree diagrams to lowest orders. We do this only for the simplest non-trivial model, namely three selfinteracting massive vector fields $\left(m_{a}>0, a=1,2,3\right)$ as in section 2. We make the same Ansatz for $W$ as in section 2 , but without the quadrilinear terms. Up to divergence- and coboundary couplings (39) this is the most general trilinear Ansatz which fulfills the requirements (a)-(e) of section 2 .

The parameters in the Ansatz for $W$ and $\mathcal{F}_{d \text { int }}^{\mu \nu}$ (67) are determined by inserting these expressions into the physicality requirement (69). Here we only state the results:

- for $W$ we obtain precisely the same expression as in section 2,

- we have computed the parameters in $\mathcal{F}_{\text {int }}$ (which are formal power series) up to second order in $g$

$$
\begin{aligned}
& \mathcal{F}_{d \text { int }}^{\mu \nu}=F_{d \text { int }}^{\mu \nu}-\frac{g}{m} \epsilon_{d b c}\left(F_{b}^{\mu \nu} \phi_{c}\right)_{\mathrm{int}}+ \frac{g}{m}\left(F_{d}^{\mu \nu} H\right)_{\mathrm{int}} \\
&-\frac{g^{2}}{4 m^{2}}\left[\delta_{d a} \delta_{b c}-\left(\delta_{d c} \delta_{b a}+\delta_{d b} \delta_{c a}\right)\right]\left(: \quad F_{a}^{\mu \nu} \phi_{b} \phi_{c}:\right)_{\mathrm{int}} \\
&-\frac{g^{2}}{2 m^{2}} \epsilon_{d b c}\left(F_{b}^{\mu \nu} \phi_{c} H\right)_{\mathrm{int}}+\frac{g^{2}}{4 m^{2}} \quad\left(: \quad F_{d}^{\mu \nu} H^{2}:\right)_{\mathrm{int}}+\mathcal{O}\left(g^{3}\right) .
\end{aligned}
$$

To get a better understanding of the latter result we identify these physical fields $\mathcal{F}_{d \text { int }}^{\mu \nu}(70)$ as gauge invariant fields in the framework of spontaneous symmetry breaking of the $S U(2)$ gauge symmetry. In this semiclassical picture the scalar fields $\phi_{a}$ (35) and $H$ (37) form two $S U(2)$ doublets

$$
\Phi=\frac{1}{\sqrt{2}}\left(\begin{array}{c}
\phi_{2}+i \phi_{1} \\
v+H-i \phi_{3}
\end{array}\right)
$$

and

$$
\tilde{\Phi}=\frac{1}{\sqrt{2}}\left(\begin{array}{c}
v+H+i \phi_{3} \\
-\phi_{2}+i \phi_{1}
\end{array}\right)
$$


where $v$ is the vacuum expectation value of the original (i.e. non-shifted) field $\tilde{H}=v+H . v$ is proportional to the gauge boson mass

$$
m=\frac{g v}{2} .
$$

The composite fields

$$
\mathcal{G}_{3}^{\mu \nu}:=\tilde{\Phi}^{*} F^{\mu \nu} \tilde{\Phi}=-\Phi^{*} F^{\mu \nu} \Phi=-\sum_{a=1,2,3} F_{a}^{\mu \nu} \Phi^{*} \sigma_{a} \Phi
$$

$\left(\sigma_{a}\right.$ are the Pauli matrices) and

$$
\mathcal{G}_{1}^{\mu \nu}:=\frac{1}{2}\left(\tilde{\Phi}^{*} F^{\mu \nu} \Phi+\Phi^{*} F^{\mu \nu} \tilde{\Phi}\right), \quad \mathcal{G}_{2}^{\mu \nu}:=\frac{i}{2}\left(\tilde{\Phi}^{*} F^{\mu \nu} \Phi-\Phi^{*} F^{\mu \nu} \tilde{\Phi}\right)
$$

are $S U(2)$ gauge invariant, or equivalently they are invariant with respect to the classical ${ }^{34}$ BRST-transformation

$s\left(F_{a}^{\mu \nu}\right)=i g \epsilon_{a b c} F_{b}^{\mu \nu} u_{c}, \quad s\left(\phi_{a}\right)=i m u_{a}+\frac{i g}{2}\left(u_{a} H+\epsilon_{a b c} \phi_{b} u_{c}\right), \quad s(H)=-\frac{i g}{2} u_{a} \phi_{a}$.

By multiplying out the matrices in $\mathcal{G}_{d}^{\mu \nu}(74,75)$ we find that (with a suitable normalization) the corresponding quantum fields are proportional to $\mathcal{F}_{d \text { int }}^{\mu \nu}$, so far as we have determined the constant power series $t_{d a b}, \ldots, \tilde{z}_{d a}$ (67), i.e.

$$
\mathcal{G}_{d \text { int }}^{\mu \nu}(x ; W)=\frac{v^{2}}{2} \mathcal{F}_{d \text { int }}^{\mu \nu}(x ; W)+\mathcal{O}\left(g^{3}\right)
$$

Finally some more remarks on the validity of the Schwinger-Swieca screening property are in order. Since the physical field strength is an operator in Hilbert space (positive definite metric) which fulfills a Maxwell type equation and since the particle spectrum has an isolated mass hyperboloid for the vector meson, the theorem of Swieca 43] is applicable and hence the charge defined in terms of the large surface integral over the physical field strength vanishes. This charge screening is more interesting if additional spinor matter is present.

\footnotetext{
${ }^{34}$ To avoid problems of defining products of interacting fields (this can be done by means of (58)) and the BRST-transformation thereof, we only consider classical fields here.
} 


\section{$5 \quad$ Renormalizability for $\mathrm{s} \geq 1$ without Ghosts?}

We have seen that the cohomological representation of the Wigner theory for vector mesons achieves the magic trick of rescuing renormalizability, whereas the naive application of the standard causal perturbation theory based on interactions $W_{\text {phys }}$ in terms of local Wick polynomials in physical (Wigner) field coordinates, which inevitably leads to dim $W_{\text {phys }} \geq 5$, fails on the count of renormalizability way which is reminiscent of a catalyzer in chemistry. This is to say a physical problem of perturbatively coupled massive spin $=1$ interactions, which a priori has nothing to do with ghosts, had to be cohomologically extended, because that was apparently the only way to reconcile the standard perturbative machinery (of deformation of free theories by $W^{\prime} s$ ) with the short distance renormalizability requirement. But at the end, after the cohomological descend, one obtains local physical vector meson fields and a physical $S$-matrix (both renormalizable) within a physical Fock space of massive spin one particles, together with new physical degrees of freedom. This is a physical result which totally conceals the intermediate presence of ghosts. Although the final result agrees formally with (the gauge invariant part of) renormalized gauge theory, the underlying physical idea and the words used to describe it are quite different. Instead of the Higgs mechanism, which generates the $\mathrm{s}=1$ mass through an additionally introduced (by hand) scalar field with non-vanishing vacuum expectation value, and which has no visible intrinsic (gauge-invariant) meaning, the vector meson mass in the present approach is directly linked with the aforementioned Schwinger-Swieca screening mechanism. The latter, together with the renormalizability requirement, demands the presence of additional degrees of freedom which we realized as scalar particles and identified with the Higgs field without its vacuum condensate.

Although we have neither demonstrated uniqueness of the ghosts nor of the new physical degree of freedom, we believe, that as already mentioned in the third section, our minimal solution is unique in the sense that any other solution involving higher spin ghost and induced physical objects always contains our minimal solution (plus possible additional couplings with coupling parameters which may be set to zero).

\footnotetext{
${ }^{35}$ This is a well-known limitation in Weinberg's program of using Wigner's particle theory as a starting point for perturbative renormalization for massive vector mesons and higher spin particles.
} 
Between the two formulations for interacting vector mesons, once as the quantized version of classical gauge theories with Higgs mechanism and on the other hand the "ghost catalyzer" to implement perturbative renormalization we prefer the latter because it underlines the preliminary aspect of our understanding of higher spin interactions more clearly. In this sense it upholds the Bohr-Heisenberg maxim that even in cases of discoveries via nonobservable and formal constructs, one should always aim for a reduction to observable concepts in order to obtain a conceptual profound understanding.

The present formulation also gives a more detailed picture of physical fields (cf. (70) ). Whereas their locality and spectral properties are as in a renormalizable lower spin $\mathrm{s}<1$ coupling, their operator dimension generally does not have the form of logarithmic corrections on canonical values of Lagrangian fields. Rather they involve higher Wick-polynomials up to operator dimension 4 i.e. are composites in the unphysical fields which interpolate "elementary" (in the sense of a perturbative description only) physical particles. This is the consequence of the fact that the cohomological descend renders the standard formulas in terms of retarded $R$-products invalid. Instead of Lagrangian interpolating fields with their canonical dimensions and logarithmic radiative corrections we have to work with the physical composites for the interpolation of the physical particles. The $Q$-invariant linear combinations which represent the physical fields within the extended Wick formalism have no intrinsic meaning or in other words the present formalism has not supplied us with a formalism which leads to the physical vacuum expectation values in a more direct and less ad hoc manner. Whereas symmetries of a free field theory can be used for the selection of a natural invariant subalgebra, the selection of a $Q$-invariant subalgebra of Wick-polynomials remains a rather unnatural procedure. Whereas an observable subalgebra resulting as the fixpoint algebra of an internal symmetry contains all the structure which via the superselection theory allows an intrinsic reconstruction of the algebra of fields, there is no intrinsic natural way to reconstruct the ghost extended algebra from the physical algebra. It also indicates that even though the final physical degrees of freedom are local and can be described in terms of covariant point-like fields, the formalism deviates in "some way" from the standard local behavior in that the perturbation cannot be interpreted as a deformation on the original local class (Borchers class) of local physical fields but rather appears as a subclass of a deformed unphysical extended theory with artificial looking rules concerning its position within the extended local class. In this way the violation of the Bohr-Heisenberg maxim about ob- 
servables in the present description of higher spin interaction becomes most evident. Before we present some ideas about an alternative approach avoiding non-observable aspects, it is helpful to recall how the Wigner theory deals with the vector potential in the case of zero mass i.e. for photons.

On a very formal level (ignoring regularisations of line integrals) we could in fact also have obtained the lowering of the operator $\operatorname{dimension} \operatorname{dim} A$ from two to one without any cohomological extension by allowing non-point-like and non-covariant vector potentials which are interpolating the same particles as the covariant ones. Although on-shell objects as the $S$-matrix of the final QFT are unaffected, as long as those fields remain almost local in the sense of [21], it is not known how to deal with such objects in a causal approach based on interaction polynomials $W$ and transition operators $S(g)$.

It is interesting to note that the Wigner theory of free photons does not allow the introduction of any local covariant vector potential, thus raising the suspicion that massless spin one interactions formulated in a physical Hilbert space lead to some sort of clash between renormalizability (which requires vector potentials of $\operatorname{dim} A_{\mu}=1$ ) and the locality and covariance of interaction densities $\mathrm{W}$. This problem with vector potentials comes from the non-compact structure of the little group (stability group) of a light-like vector say $p_{R}=(1,0,0,1)$, which is the twofold covering of the Euclidean group $E(2)$ (and is denoted by $\tilde{E}(2)$ ). Whereas the rotation has the interpretation as a helicity rotation (rotation around the third axis), the "translations" are Lorentz-transformations which tilt the t-z wedge, leaving its upper light-like vector unchanged. As far as the two transversal coordinates are concerned they behave like 2-parametric Galilei velocity transformations (i.e. "light cone translations" without the energy positivity property) with the two longitudinal light cone translations playing the role of the Hamiltonian resp. the central mass in the quantum mechanical representation theory of the Galilei group. The embedding of $\tilde{E}(2)$ into $S L(2, \mathbf{C})$ for the above choice of reference vector is

$$
\alpha(\rho, \theta)=\left(\begin{array}{ll}
e^{i \frac{1}{2} \theta} & \rho \\
0 & e^{-i \frac{1}{2} \theta}
\end{array}\right), p_{R} \sim\left(\begin{array}{ll}
2 & 0 \\
0 & 0
\end{array}\right),
$$

where $\theta$ is the angle for the rotations around the 3 -axis and $\rho=\rho_{1}+i \rho_{2}$ parameterizes the Euclidean translations by the vector $\left(\rho_{1}, \rho_{2}\right)$. The unitary representation theory of this non-compact group is somewhat more complicated than that of $S U(2)$. But it is obvious that the representations fall 
into two classes; the "neutrino-photon" class with $U(\alpha(\rho, 0))=1$ i.e. trivial representation of the Euclidean translations, and the remaining faithful "continuous spin" (infinite dimensional) representations with $U(\alpha(\rho, 0)) \neq 1$. A more detailed analysis shows that the latter lacks the strong localization requirements which one must impose on those positive energy representations which are used for the description of particles. ${ }^{0}$ Hence the Wigner theory does not allow to describe photon operators in terms of covariant vector fields. On the other hand a covariant field strength $F_{\mu \nu}$ has the following intertwiner representation in terms of the Wigner annihilation/creation operators for circular polarized photons $a_{ \pm}^{\#}(k)$

$$
\begin{aligned}
& F_{\mu \nu}(x)=\frac{1}{(2 \pi)^{\frac{3}{2}}} \int\left\{e^{-i k x} \sum_{ \pm} u_{\mu \nu}^{( \pm)}(k) a_{ \pm}(k)+h . c .\right\} \frac{d^{3} k}{2|k|} \\
& u_{\mu \nu}^{( \pm)}(k) \simeq k_{\mu} e_{\nu}^{( \pm)}(k)-k_{\nu} e_{\mu}^{( \pm)}(k)
\end{aligned}
$$

Here $e_{\mu}^{( \pm)}(k)$ are the polarization vectors which are obtained by application of the following Lorentz transformation to the standard reference vectors $\frac{1}{\sqrt{2}}(0, \pm 1, i, 0)$ (which are $\left.\perp(1,0,0,1)\right)$ : a rotation of the $z$-axis into the momentum direction $\vec{n} \equiv \frac{\vec{k}}{\omega}$ (fixed uniquely by the standard prescription in terms of two Euler angles) and a subsequent Lorentz-boost along this direction which transforms $(1, \vec{n})$ into $k=\omega(1, \vec{n})$. It is these vectors that do not behave covariant under those Lorentz-transformations which involve the above "little group translations" but rather produce an affine transformation law

$$
G(\rho) e^{(\lambda)}\left(p_{R}\right)=e^{(\lambda)}\left(p_{R}\right)+\left\{\begin{array}{ll}
-\frac{1}{2}(\bar{\rho}, 0,0, \bar{\rho}), & \lambda=+ \\
+\frac{1}{2}(\rho, 0,0, \rho), & \lambda=-
\end{array} \quad \rho=\rho_{1}+i \rho_{2}\right.
$$

$(G(\rho)$ is the Minkowski space representation of the Euclidean translations by $\left.\left(\rho_{1}, \rho_{2}\right)\right)$ whereas under $x-y$ rotations the $e^{(\lambda)}$ picks up the standard Wigner phase factor. The polarization vectors do not behave as 4 -vectors since they are not invariant under the Euclidean translations in $\tilde{E}(2)$, as one would have expected for a (non-existing!) bona fide intertwiner from the $(0, h=1)$

\footnotetext{
${ }^{36}$ For the non-faithful representation $(m=0, h=$ semi-integer $)(U(\alpha(\rho, 0)) \neq 1)$ one finds that the infinite set of intertwiners in (52) is restricted to $u^{\left(n_{+}, n_{-}\right)}$with $n_{-}=n_{+} \pm h$ 46.
} 
Wigner representation to the $D^{\left[\frac{1}{2}, \frac{1}{2}\right]}$ covariant representation. Rather the intertwiner only has Lorentz-covariance up to additive gauge transformations i.e. up to affine longitudinal terms. For general Lorentz transformations this affine law reads:

$$
(U(\Lambda) e)_{\mu}(k)=\Lambda_{\mu}^{\nu} e_{\nu}\left(\Lambda^{-1} k\right)+k_{\mu} H(\Lambda, k)
$$

This peculiar manifestation of the $(0, h=1)$ little group $\tilde{E}(2)$ is the cause for the appearance of the local gauge issue in local quantum physics. Unfortunately this quantum origin is somewhat hidden in the quantization approach, where it remains invisible behind the geometrical interpretation in terms of fibre bundles. In terms of the potential in the physical Fock space we have

$$
U(\Lambda) A_{\mu}(x) U^{*}(\Lambda)=\Lambda_{\mu}^{-1 \nu} A_{\nu}(\Lambda x)+\partial_{\mu} H
$$

where $H$ is a concrete operator involving the $a_{ \pm}^{\#}$ and $e_{ \pm}$from (80). Formally the string-like localized vector potential (with localization chosen in the space-like $n$-direction) may be written as a line integral over the field strength

$$
\begin{aligned}
& \partial_{\mu} A_{\nu}(x)-\partial_{\nu} A_{\mu}(x)=F_{\mu \nu}(x) \\
& A_{\nu}=\frac{1}{n \cdot \partial} n^{\mu} F_{\mu \nu} \curvearrowright n \cdot A=0 .
\end{aligned}
$$

The regularization needed in order to make mathematical sense out of these string-like objects and related technical problems will not be discussed in this qualitative presentation. The physical manifestation of the existence of the somewhat nonlocal vector potential is the breakdown of the additivity of the algebras generated by the field strength for non-simply connected regions [21]. The natural localization regions for vector potentials are space-like cones or wedges which contain such cones. On the other hand the causal perturbation theory (and any other formulation using Lagrangian quantization) requires point-like fields.

Comparing this with the massive case, one notes that the problematic aspects of the use of vector potentials in the local description of $s=1$ show up in the quantum properties of free Wigner photons before the renormalization procedure, whereas for massive vector mesons it appears only in facing the issue of renormalizability (dimensional counting) of interactions. What is in common to both cases is the fact that no existing formulation of renormalized 
perturbation theory is capable to deal with $\operatorname{dim} A_{\mu}=1$ nonlocal vector potentials within the framework of causal Wick-polynomials $W$. Both cases can be dealt with in terms of the same remedy namely cohomological extension by ghosts within a BRS-like formalism; however as a result of the availability of arguments based on scattering theory, the BRS formalism is conceptually much simpler in the massive case, whereas in the massless theory one must use the full BRS-formalism which suffers contribution from interactions and requires additional concepts for the separation of observable algebras from physical states [10].

It should be clear from these remarks that in order to follow the BohrHeisenberg maxim and remove the "ghost-catalyzers" in favor of a ghostfree formulation, one has to go significantly outside the present perturbative framework. A hint where to look comes from the observation that the ghosts have been introduced to lower the operator dimension of $A_{\mu}$ from two to one, while still maintaining the (formal) locality and the covariance of the operators. This is clearly an off-shell short distance argument. So this observation suggests to look for an on-shell formulation. On-shell quantities are the true S-matrix and formfactors of physical operators $A$ between multiparticle scattering states:

$$
\begin{aligned}
& S\left|p_{1}, \ldots p_{n}\right\rangle^{\text {out }}=\left|p_{1}, \ldots p_{n}\right\rangle^{\text {in }} \\
& { }_{\text {out }}\left\langle p_{1}^{\prime} \ldots p_{m}^{\prime}|A| p_{1}, \ldots p_{n}\right\rangle^{\text {in }}
\end{aligned}
$$

Note that we defined the formfactors in such a way that the matrix elements of $S$ themselves correspond to the formfactor of the identity operator. So the first question is: can one compute the perturbative expansion of $S$ while staying on-shell all the time. The causal approach used before does not fulfill this requirement, since the Bogoliubov transition operator $S(g)$ is off shell (in the sense of this paper) and only approaches the mass shell in the adiabatic limit57. In fact it is not possible to formulate causality which involves products of fields directly on-shell, rather the on-shell substitute is the notoriously elusive crossing symmetry together with some inexorably linked subtle analytic continuation properties. So the question is: does there exist a

\footnotetext{
${ }^{37}$ The difference between on- and off-shell looks quite innocuous in momentum space, however the spacetime aspects could be different. In order to illustrate this point, just look at the massive Thirring model. The $S(g)$ resp. the field correlation functions show the full field theoretic vacuum- and one-particle polarization structure (virtual particle structure) whereas the $S$-matrix and the closely related PFG wedge generators (which are introduced below) are "quantum mechanical" i.e. obey particle number conservation.
} 
formulation of quantum field theory which, different from quantization, uses such on-shell concepts in its construction.

Fortunately the beginning of such a new approach to QFT already exists [32] 36]. This approach can be viewed as a generalization of the Wigner theory in the presence of interactions. It bypasses completely the use of point-like fields and aims directly at the wedge algebras and the associated net of smaller localized algebras obtained by intersecting wedge algebras.

It is deeply satisfying that the modular theory used in this construction directly converts the Wigner one particle theory into the interaction free nets of algebras without using fields and their equivalence classes in intermediate steps. The generalization to interacting models is given by the modular interpretation of the S-matrix as a relative modular invariant which "measures" the interaction for wedge-localized algebras. The naturalness of the wedge region appeared for the first time long ago in Unruh's Minkowski space analogue of the Hawking thermal aspects of black holes. Indeed, its use for a generalization of the Wigner approach in the presence of interactions leads rather swiftly to a profound understanding of two aspects: the relation of the thermal aspect of modular (wedge) localization with the aforementioned elusive crossing symmetry of particle physics, and the existence of generators of the wedge algebra which, applied to the vacuum, create one particle states free of particle-antiparticle polarization clouds, despite the presence of interactions. The crossing is known to be closely related to the TCP theorem; in fact it is a kind of TCP property for individual particle in formfactors whereby the particle in an incoming configuration is flipped into an (analytically continued) outgoing anti-particle. The existence of (vacuum) polarization free generators (abbreviated "PFG") for wedge localized algebras on the other hand is a new discovery. Their correlation functions can be expressed in terms of "nested" products of S-matrices [36]. A closer look has revealed that in the special case of $\mathrm{d}=1+1$ purely elastic S-matrices ("factorizing models" [24] [39]) the PFG's in fact obey the Zamolodchikov-Faddeev algebra, and that the latter receives in turn for the first time a physical spacetime interpretation which transcends its formal use [33] 34] 35] 36].

The potential relevance of these remarks for a ghostfree formulation of higher spin interactions becomes clearer if one notices that the reasons for their introduction is that the point-like interaction densities $W_{\text {phys }}$ in terms of physical higher spin fields have a short distance behavior beyond the one allowed by the formal renormalizability criterion. If we could avoid such off-shell objects as point-like fields and their correlation functions in favor 
of formfactors of localized operators (85) then the existence of the theory would not be threatened by the short distance behavior in the construction of the theory. Rather it would come out from the definition of formfactors of products of such operators by summing over the complete set of intermediate particle states. The first step in such a new construction program namely the construction of the PFG's belonging to the model is purely on-shell and only involves physical particles. The correlation functions of PFG's are on-shell objects, in the old fashioned language they contain a "natural cutoff" as a result of their somewhat de-localized nature, but unlike the brutal force cutoffs in the Lagrangian approach, these objects coexist in the same Hilbert space together with sharper localized operators which belong to intersections of wedge algebras. The non-triviality check in this approach, which is expected to replace the good short distance behavior in the standard renormalization theory, is the non-triviality of algebras localized in double cones obtained by intersecting wedges

$$
\mathcal{A}(\mathcal{O})=\cap_{\mathcal{O} \subset W} \mathcal{A}(W)
$$

It may not be widespread known, but it is nevertheless true that all physical informations are contained in local algebras $\$ 8$ and that there is no necessity from a conceptual point of view to use point-like field coordinates apart from certain distinguished point-like currents related to symmetries and possibly associated order/disorder fields.

The first step, namely the construction of the on-shell PFG's is reminiscent of attempts in the $60^{i e s}$ to construct unitary crossing symmetric S-matrices. This attempt, even in its limited perturbation version, was illfated and did not lead to tangible results. It remained as a very fundamental problem in particle physics closely related to the inverse problem i.e. the question to what extend a physically admissible (on-shell) S-matrix determines uniquely an (off-shell) algebraic net. The modular approach to wedge algebras and the concept of PFG's incorporates and enriches this old S-matrix program, so that it becomes the first step in a construction of local nets. The hope that in this new version it becomes at least susceptible to a kind of onshell perturbation theory rests on the new field theoretic setting together with rich new concepts which were absent in the days of the old bootstrap program. In fact the success of this program for $d=1+1$ factorizing models

\footnotetext{
${ }^{38}$ This independence of the physical content from field coordinatizations is the main reason for the interest in the algebraic approach (i.e. local quantum physics).
} 
[24] 39] (in which case such an S-matrix which avoids the standard construction exists even in a summed up non-perturbative form) may be taken as an encouragement for a future exploration of this new approach to QFT.

One expects that this new approach reproduces the perturbative results of the standard renormalization theory wherever the latter is applicable. The innovative power of the new approach is expected to show up in situations where one has reasons to believe that Lagrangian quantization does not provide physical field coordinates of sufficient low operator dimension related to good short distance properties needed for the W's in the causal approach. There is no a priori reason why the Lagrangian fields should be the ones with the lowest short distance dimensions in the local equivalence class of all local fields of one model. In the case of spin $=1$ one can still overcome such obstacles against the standard formulations by the ad hoc ghost trick which is suggested by the quantization of gauge theories. But could there also be (e.g. higher spin) cases with a finite number of coupling parameters which are declared "non-renormalizable" in the standard approach, even though they have a well-defined perturbation theory in the new sense?

\section{$6 \quad$ Future Perspectives}

The history of gauge theory and its connection with higher spin renormalizable QFT is one of the theoretically most fascinating and experimentally most significant developments in the $20^{\text {th }}$ century. Contrary to a widely held opinion this is still an unfinished story with possible future surprises.

The usefulness of the role of the "gauge principle" as a selection principle in the classical setting and its attractive geometric appeal in the semiclassical realm of quantum matter coupled to external fields (minimal electromagneticcoupling) is universally recognized. Acknowledging its important role in the discovery of the renormalization of interactions between massive vector mesons, we nevertheless tried to argue in this paper that further progress in this area requires new ideas beyond those of standard gauge theory, which are of a more algebraic (or local quantum physical) than differential geometric kind. As a compromise between the standard approach and a future ghostfree framework we presented a formulation of interactions of massive vector mesons using ghosts which extend the Wigner one-particle states of the vector meson. In this formulation the necessity of the presence of further Higgs like physical degrees of freedom is most easily recognizable, even though 
they have been stripped of their role of "fattening" the photons/gluons into massive vector mesons via Higgs condensates since their mass was assumed non-vanishing from the outset. It is interesting to note that even without this role their presence is nevertheless necessary for perturbative consistency and the Schwinger-Swieca charge screening mechanism. Although we hope that this formulation will only be intermediary and replaced eventually by a ghostfree modular approach (the remarks in the previous section), it is in our view already with all its shortcomings better suitable than the standard gauge approach to highlight those points which suggest the necessity of a radically different formulation.

The viewpoint in this paper is in some ways opposite to the standard one. Whereas standardly the massless case is used as the basic reference and the mass of vector mesons is attributed to the Higgs mechanism in gauge theories which follows more the geometric logic of classical field theory, we have favored a particle viewpoint which emphasizes the physical particle interpretation and leaves the more difficult off-shell infrared rearrangements in the massless limit to future more detailed investigations. Whether a completely ghostfree formulation (which fully explains the gauge-restricted structure of interacting higher spin objects as consequences of the same basic local quantum physical properties which hold for low spins) is feasible, only future can tell.

We also mentioned various historical precedents to our point of view. In this context it is interesting to add a quotation from a paper of P.W Anderson [1]. About the possible non-intrinsic aspect of the Higgs mechanism for massive photons in comparison to plasmons he writes: "How, then, if we were confined to a plasma as we are to the vacuum and could only measure renormalized quantities, might we try to determine whether, before turning on the effects of electromagnetic interaction, $A$ (the vector potential) had been a massless gauge field and... ? As far as we can see this is not possible." The lack of intrinsic physical meaning of Higgs condensates was also the main issue of the well-known Elitzur's theorem.

If by our observations on massive vector mesons we succeed to re-direct some of the attention which has been given to geometric/mathematical aspects towards the many open and interesting conceptual local quantum problems, the time it took for writing this paper was well spent.

\section{Acknowledgments:}

The authors thank Klaus Fredenhagen for helpful suggestions and one of us (M. D.) is indebted to Günter Scharf for several fruitful discussions. 


\section{Appendix A: The $S$-matrix to first order}

First we give an incomplete proof of the following statement (which is of general interest):

Conjecture: Let $V(x)$ be an arbitrary Wick polynomial with derivatives (i.e. an arbitrary element of the Borchers class of the free fields) and let all free fields be massive. Then the relation

$$
\lim _{\epsilon \rightarrow 0} \int d^{4} x V(x) g(\epsilon x)=0, \quad\left(g \in \mathcal{D}\left(\mathbf{R}^{4}\right), \quad g(0)=1\right)
$$

implies that there exists another Wick polynomial (with derivatives) $W_{1}^{\mu}$ such that

$$
V=\partial_{\mu} W_{1}^{\mu}+V_{0}
$$

Thereby $V_{0}$ is the sum of all Wick monomials $C: \partial^{a_{1}} A_{j_{1}}(x) \ldots \partial^{a_{n}} A_{j_{n}}(x)$ : $\left(C=\right.$ const., $A_{j}$ is a free field with mass $\left.m_{j}\right)$ in $V$ for which the masses $m_{j_{1}}, \ldots, m_{j_{n}}$ are such that the solution for $\left(k_{1}, \ldots, k_{n}\right) \in \mathbf{R}^{4 n}$ of

$$
k_{1}+\ldots+k_{n}=0 \quad \bigwedge \quad k_{l}^{2}=m_{j_{l}}^{2} \quad \forall l=1, \ldots, n
$$

contains no non-empty open subset of the manifold $k_{l}^{2}=m_{j_{l}}^{2} \quad \forall l=1, \ldots, n$.

A simple example for a term in $V_{0}$ is $: \varphi(x)^{3}:$, where $\left(\square+m^{2}\right) \varphi=0, m>$ 0 .

Incomplete proof 59 To simplify the notations we assume that the free fields are bosonic scalars, the generalization to other types of fields is obvious. Global factors $2 \pi$ are omitted in the whole proof. We write $V$ in the form

$$
V(x)=\sum_{n} \sum_{j_{1}, \ldots, j_{n}} P_{j_{1} \ldots j_{n}}\left(\partial^{x_{1}}, \ldots, \partial^{x_{n}}\right): A_{j_{1}}\left(x_{1}\right) \ldots A_{j_{n}}\left(x_{n}\right):\left.\right|_{x_{1}=\ldots=x_{n}=x}
$$

where the sum over $n$ is finite, the $A_{j}$ 's are free fields and $P_{j_{1} \ldots j_{n}}\left(\partial^{x_{1}}, \ldots, \partial^{x_{n}}\right)$ is a polynomial in the partial derivatives $\partial_{\mu}^{x_{l}}, 1 \leq l \leq n$. Obviously we may replace $P_{j_{1} \ldots j_{n}}\left(\partial^{x_{1}}, \ldots, \partial^{x_{n}}\right)$ by

$$
\bar{P}_{j_{1} \ldots j_{n}}\left(\partial^{x_{1}}, \ldots, \partial^{x_{n}}\right) \equiv \frac{1}{n !} \sum_{\pi \in \mathcal{S}_{n}} P_{j_{\pi(1)} \ldots j_{\pi(n)}}\left(\partial^{x_{\pi(1)}}, \ldots, \partial^{x_{\pi(n)}}\right)
$$

\footnotetext{
${ }^{39}$ Klaus Fredenhagen told us the main idea of proof. The method is strongly influenced by the proof of lemma 2 (including appendix (b)) in [7.
} 
in (90). In terms of annihilation and creation operators the free fields read

$$
A_{l}(y)=\int d^{4} p \delta\left(p^{2}-m_{l}^{2}\right) \bar{A}_{l}(p) e^{i p y}, \quad \bar{A}_{l}(p):=\left[\Theta\left(-p^{0}\right) a_{l}(-\vec{p})+\Theta\left(p^{0}\right) b_{l}^{+}(\vec{p})\right]
$$

$\left(a_{l}=b_{l}\right.$ is possible), where $\left[b_{l}(\vec{p}), b_{j}^{+}(\vec{k})\right]=\delta_{l j} 2 \sqrt{\vec{p}^{2}+m_{l}^{2}} \delta^{3}(\vec{p}-\vec{k})$ and similar for $\left[a_{l}, a_{j}^{+}\right]$. Now we insert (90-92) into (87)

$$
\begin{aligned}
0= & \lim _{\epsilon \rightarrow 0} \sum_{n} \int d x g(\epsilon x) \int d x_{1} \ldots d x_{n} \delta\left(x_{1}-x, \ldots, x_{n}-x\right) \cdot \\
& \cdot \sum_{j_{1}, \ldots, j_{n}} \bar{P}_{j_{1} \ldots j_{n}}\left(\partial^{x_{1}}, \ldots, \partial^{x_{n}}\right): A_{j_{1}}\left(x_{1}\right) \ldots A_{j_{n}}\left(x_{n}\right): \\
= & \sum_{n} \int d^{4} p_{1} \ldots d^{4} p_{n} \sum_{j_{1}, \ldots, j_{n}} \bar{P}_{j_{1} \ldots j_{n}}\left(i p_{1}, \ldots, i p_{n}\right) \delta^{4}\left(p_{1}+\ldots+p_{n}\right) . \\
& \cdot \prod_{l=1}^{n} \delta\left(p_{l}^{2}-m_{j_{l}}^{2}\right)\left[\Theta\left(-p_{l}^{0}\right) a_{j_{l}}\left(-\vec{p}_{l}\right)+\Theta\left(p_{l}^{0}\right) b_{j_{l}}^{+}\left(\vec{p}_{l}\right)\right]: .
\end{aligned}
$$

(Here we have exchanged the order of the integrations and the limit $\epsilon \rightarrow 0$. This can be justified by considering matrix elements between wave packets.) Taking improper matrix elements $<b_{r_{1}}^{+}\left(\vec{k}_{1}\right) \ldots b_{r_{s}}^{+}\left(\vec{k}_{s}\right) \Omega \mid \ldots a_{r_{s+1}}^{+}\left(-\vec{k}_{s+1}\right) \ldots a_{r_{t}}^{+}\left(-\vec{k}_{t}\right) \Omega>$ of (93), where $\Omega$ is the vacuum of the free fields, we obtain $\square$

$$
0=\bar{P}_{r_{1} \ldots r_{t}}\left(i k_{1}, \ldots, i k_{t}\right) \delta^{4}\left(k_{1}+\ldots+k_{t}\right) \quad \text { where } \quad k_{l}=\left( \pm \sqrt{\vec{k}_{l}^{2}+m_{r_{l}}^{2}}, \vec{k}_{l}\right) \forall l
$$

more precisely $k_{l}^{0}>0$ for $l=1, \ldots, s$ and $k_{l}^{0}<0$ for $l=s+1, \ldots, t$. If $r_{1}, \ldots, r_{t}$ are such that the corresponding term belongs to $\left(V-V_{0}\right)$, then the condition (94) restricts the polynomial $\bar{P}_{r_{1} \ldots r_{t}}$ in the following way. We make a Taylor expansion with respect to $k=\left(k_{1}+\ldots+k_{t}\right)$ at $k=0$ :

$\bar{P}_{r_{1} \ldots r_{t}}\left(i k_{1}, \ldots, i k_{t}\right)=\bar{P}_{r_{1} \ldots r_{t}}\left(i k_{1}, \ldots, i k_{t-1},-i\left(k_{1}+\ldots+k_{t-1}\right)\right)+i k_{\mu} \tilde{Q}_{r_{1} \ldots r_{t}}^{\mu}\left(k_{1}, \ldots, k_{t}\right)$

where $\tilde{Q}_{r_{1} \ldots r_{t}}^{\mu}$ is also a polynomial. From (94) we conclude (for all $\left(r_{1}, \ldots, r_{t}\right)$ which appear in $\left.\left(V-V_{0}\right)\right)$ that $\bar{P}_{r_{1} \ldots r_{t}}\left(i k_{1}, \ldots, i k_{t-1},-i\left(k_{1}+\ldots+k_{t-1}\right)\right)$ vanishes

\footnotetext{
${ }^{40}$ Usually there are several possibilities to contract the creation and annihilation operators. All give the same contribution due to the permutation symmetry of $\bar{P}_{r_{1} \ldots r_{t}}$ (91).
} 
on a non-empty open subset of the manifold $k_{i}^{2}=m_{r_{i}}^{2} \forall i=1, \ldots, t-1$ and $\left(k_{1}+\ldots+k_{t-1}\right)^{2}=m_{r_{t}}^{2}$. We strongly presume that this implies that there exist other polynomials $P^{(l)}\left(k_{1}, \ldots, k_{t-1}\right)(l=1, \ldots, t)$ (for simplicity we omit the indices $\left.r_{1}, \ldots, r_{t}\right)$ such that

$$
\begin{aligned}
\bar{P}\left(i k_{1}, \ldots, i k_{t-1},-i\left(k_{1}+\ldots k_{t-1}\right)\right) & =\sum_{l=1}^{t-1}\left(k_{l}^{2}-m_{r_{l}}^{2}\right) P^{(l)}\left(k_{1}, \ldots, k_{t-1}\right) \\
& +\left(\left(k_{1}+\ldots+k_{t-1}\right)^{2}-m_{r_{t}}^{2}\right) P^{(t)}\left(k_{1}, \ldots, k_{t}(96)\right)
\end{aligned}
$$

For vanishing masses this conjecture has been proved by Buchholz and Fredenhagen (in appendix (b) of [7]).

$$
\begin{aligned}
& \bar{P}\left(i k_{1}, \ldots, i k_{t}\right) \prod_{l=1}^{t} \delta\left(k_{l}^{2}-m_{r_{l}}^{2}\right) \quad=\quad\left[\left(\left(k_{1}+\ldots+k_{t-1}\right)^{2}-k_{t}^{2}\right) P^{(t)}\left(k_{1}, \ldots, k_{t-1}\right)\right. \\
& \left.+i k_{\mu} \tilde{Q}^{\mu}\left(k_{1}, \ldots, k_{t}\right)\right] \prod_{l=1}^{t} \delta\left(k_{l}^{2}-m_{r_{l}}^{2}\right) \\
& =i\left(k_{1}+\ldots+k_{t}\right)_{\mu} \quad Q^{\mu}\left(i k_{1}, \ldots, i k_{t}\right) \prod_{l=1}^{t} \delta\left(k_{l}^{2}-m_{r_{l}}^{2}\right),
\end{aligned}
$$

where $Q^{\mu}\left(i k_{1}, \ldots, i k_{t}\right):=-i\left(k_{1}+\ldots+k_{t-1}-k_{t}\right)^{\mu} P^{(t)}\left(k_{1}, \ldots, k_{t-1}\right)+\tilde{Q}^{\mu}\left(k_{1}, \ldots, k_{t}\right)$. Next we symmetrize $Q^{\mu}$ according to (91) and denote the result by $\bar{Q}^{\mu}$. The equation (97) holds still true if we replace $Q^{\mu}$ by $\bar{Q}^{\mu}$. Summing up we obtain

$$
\begin{aligned}
\left(V-V_{0}\right)(x)= & \sum_{n} \sum_{j_{1}, \ldots, j_{n}} \int d^{4} p_{1} \ldots d^{4} p_{n} i\left(p_{1}+\ldots+p_{n}\right)_{\mu} \bar{Q}_{j_{1} \ldots j_{n}}^{\mu}\left(i p_{1}, \ldots, i p_{n}\right) \cdot \\
& \cdot e^{i\left(p_{1}+\ldots+p_{n}\right) x}: \prod_{l=1}^{n} \delta\left(p_{l}^{2}-m_{j_{l}}^{2}\right)\left[\Theta\left(-p_{l}^{0}\right) a_{j_{l}}\left(-\vec{p}_{l}\right)+\Theta\left(p_{l}^{0}\right) b_{j_{l}}^{+}\left(\vec{p}_{l}\right)\right]: \\
= & \partial_{\mu}^{x} \sum_{n} \sum_{j_{1}, \ldots, j_{n}} \bar{Q}_{j_{1} \ldots j_{n}}^{\mu}\left(\partial^{x_{1}}, \ldots, \partial^{x_{n}}\right): A_{j_{1}}\left(x_{1}\right) \ldots A_{j_{n}}\left(x_{n}\right):\left.\right|_{x_{1}=\ldots=x_{n}=x}
\end{aligned}
$$

(we set $\bar{Q}_{r_{1} \ldots r_{t}}^{\mu}:=0$ if $\left(r_{1}, \ldots, r_{t}\right)$ belongs to $\left.V_{0}\right)$ which is the assertion (88).

\footnotetext{
${ }^{41}$ Unfortunately a straightforward generalization of their proof does not work. However, for our purpose we do not need the validity of (96).
} 
We do not need the general result (87-89), we only deal with very special situations. Physical consistency (31) to first order means

$$
\left.\lim _{\epsilon \rightarrow 0} \int d^{4} x g(\epsilon x)[Q, W(x)]\right|_{\operatorname{ker} Q}=0 .
$$

Let us first consider the quadrilinear terms in $V(x):=[Q, W(x)]$, i.e. $\left[Q, W(x)^{(4)}\right]$,

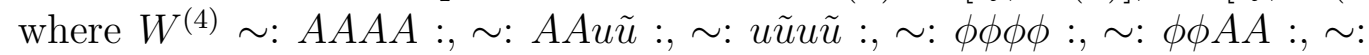
$\phi \phi u \tilde{u}:, \sim: \phi \phi H^{2}:, \sim: A A H^{2}:, \sim: u \tilde{u} H^{2}:, \sim: H^{4}:$ (the upper index (4) refers to the quadrilinear terms). They satisfy the kinematical equations (89) for a sufficient big set of momenta, i.e. they belong to $\left(V-V_{0}\right)$.t2 The polynomials $P_{j_{1} \ldots j_{n}}$ (90) (and hence also $\bar{P}(91)$ ) are of the form

$$
\bar{P}\left(i k_{1}, \ldots, i k_{4}\right)=c_{0} \quad \text { or } \quad \bar{P}^{\mu}\left(i k_{1}, \ldots, i k_{4}\right)=\sum_{i=1}^{4} c_{i} k_{i}^{\mu}
$$

where $c_{0}$ and $c_{i}$ are constants. Taking matrix elements of (98) with improper two-particle states, where the incoming state is an element of $\operatorname{ker} Q$, we obtain (94), which implies $\bar{P}=0$ or $\bar{P}^{\mu}=c\left(k_{1}^{\mu}+\ldots+k_{4}^{\mu}\right)(c=$ constant). Hence (98) implies that there exists a Wick polynomial $W_{1}^{(4) \nu}$ such that

$$
\left[Q, W(x)^{(4)}\right]=i \partial_{\nu} W_{1}^{(4) \nu}(x)
$$

This is the Q-divergence condition to first order (29). In addition $W^{(4)}$ must satisfy the requirements (a)-(e) listed in section 2. The only solution is $\sim: H^{4}:$ 20, but such a term will be excluded by physical consistency (31) to second order.

A trilinear term in $W(x)$ (with masses $m_{1}, m_{2}$ and $\left.m_{3}\right)$ fulfills the kinematical equations (89) for a sufficient big set iff there exists a permutation $\pi \in \mathcal{S}_{3}$ such that

$$
m_{\pi(3)}>m_{\pi(1)}+m_{\pi(2)}
$$

But physical consistency to higher orders will require $m_{1}=m_{2}=m_{3}=: \mathrm{m}$ apart from the $H$-couplings $\left(m_{H}>0\right.$ will not be restricted; see sect. 2).

\footnotetext{
${ }^{42}$ For a free field $\varphi$ note that $[Q, \varphi(x)]$ satisfies the same field equation as $\varphi$ and hence has the same mass.
} 
Neglecting this exception we conclude from (93) that the trilinear part $S_{1}^{(3)}$ of the $S$-matrix to first order vanishes in the adiabatic limit, i.e.

$$
S_{1}^{(3)}=\lim _{\epsilon \rightarrow 0} \int d^{4} x g(\epsilon x) W^{(3)}(x)=0,
$$

and $\left[Q, S_{1}^{(3)}\right]=0$ (31) is trivially satisfied. To get restrictions on $W^{(3)}$ we must consider physical consistency (31) to orders $n \geq 2$, especially for second order tree diagrams.

\section{Appendix B: Determination of the parame- ters in $W$ and $\mathcal{F}_{\text {int }}$ by requiring $\left[Q, \mathcal{F}_{\text {int }}(x ; W)\right]=$ 0}

The calculation is lengthy, hence we concentrate on the essential points. We determined the parameters in the Ansatz for $W$ (41) and $\mathcal{F}_{\text {int }}$ (67) by inserting these expressions into the physicality requirement (69) to lowest perturbative orders. Once a parameter is fixed, its value will be used in the following calculations without mentioning it.

- To zeroth order the condition is trivially fulfilled due tot3

$$
\left[Q, F_{d}^{\mu \nu}\right]=0
$$

- To first order the equation

$$
\left[Q, \mathcal{F}_{d}^{(1)}(x)\right]+i \int d x_{1}\left[Q, R\left(W\left(x_{1}\right) ; F_{d}(x)\right)\right]=0
$$

is required. The second term contains tree diagrams only. By means of (60) they can be written in the form

$$
\begin{aligned}
R\left(W\left(x_{1}\right) ; F_{d}^{\mu \nu}(x)\right) & =\Theta\left(x^{0}-x_{1}^{0}\right)\left[F_{d}^{\mu \nu}(x), W\left(x_{1}\right)\right] \\
+\delta\left(x-x_{1}\right) i C \frac{1}{2}\left(f_{b c d}-f_{c b d}\right) & : \quad A_{b}^{\mu}\left(x_{1}\right) A_{c}^{\nu}\left(x_{1}\right):
\end{aligned}
$$

\footnotetext{
${ }^{43}$ If we would not have made the assumption $c_{k}^{(0)}=0$ (i), we would find here that most of the zeroth order coefficients must vanish. It would remain $\mathcal{F}_{d}^{\mu \nu(0)}=F_{d}^{\mu \nu}+x_{d a}^{(0)} F_{a}^{\mu \nu} H+z_{d a}^{(0)}$ : $F_{a}^{\mu \nu} H^{2}$ : only. $x^{(0)}$ and $z^{(0)}$ are then forced to vanish by higher orders of (69). But this complicates the calculation a lot and the assumption $c_{k}^{(0)}=0$ is reasonable. So we do not do this effort here.
} 
where $C$ is an arbitrary constant. The terms $\sim \Theta\left(x^{0}-x_{1}^{0}\right)$ have propagators $\Delta^{\text {ret }}\left(x-x_{1}\right), \partial \Delta^{\text {ret }}\left(x-x_{1}\right), \partial \partial \Delta^{\text {ret }}\left(x-x_{1}\right)$, where $\Delta^{\text {ret }}$ is the retarded fundamental solution of the Klein Gordon equation, supp $\Delta^{\text {ret }} \subset \bar{V}^{+}$. The term $\sim$ $C \delta\left(x-x_{1}\right)$ is due to the non-uniqueness of the extension of $T\left(W\left(x_{1}\right) F_{d}^{\mu \nu}(x)\right)$ to the diagonal $x_{1}=x_{2}$. This is similar to the tree normalization terms (49). The tensor $f_{b c d}$ which is the same as in the Ansatz for $W^{\text {ma }}$ is antisymmetrized according to the normalization condition $\mathcal{F}_{d \text { int }}^{\mu \nu}=-\mathcal{F}_{d \text { int }}^{\nu \mu}$ (iv). Now let $\psi_{1}, \psi_{2}$ be arbitrary free fields and $y_{1}, y_{2} \in \mathbf{R}^{4},\left(y_{j}-x\right)^{2}<0(j=1,2)$. By commuting (102) with $\psi_{1}\left(y_{1}\right)$ and $\psi_{2}\left(y_{2}\right)$ we obtain

$$
0=\int d x_{1}\left[\left[\left[Q,\left[F_{d}(x), W\left(x_{1}\right)\right]\right], \psi_{1}\left(y_{1}\right)\right], \psi_{2}\left(y_{2}\right)\right] \Theta\left(x^{0}-x_{1}^{0}\right) .
$$

We conclude that for $x_{1} \in\left(x+\bar{V}^{-}\right) \backslash\{x\}$ the expression $\left[Q,\left[F_{d}(x), W\left(x_{1}\right)\right]\right]$ must be a divergence of a local operator with respect to $x_{1}$, th $^{2}$ i.e. there must exist a two-point distribution $R_{1 d}^{\tau}\left(x_{1}, x\right)$ with

$$
\Theta\left(x^{0}-x_{1}^{0}\right)\left[Q,\left[F_{d}(x), W\left(x_{1}\right)\right]\right]=\partial_{\tau}^{x_{1}} R_{1 d}^{\tau}\left(x_{1}, x\right)+\delta\left(x-x_{1}\right) P_{d}(x),
$$

where $P_{d}(x)$ is a Wick polynomial (with constant coefficients) in free fields (possibly with derivative). In contrast to the adiabatic limit of $W$ (87) there is no kinematical restriction (in the sense of (89)) here, because one of the free field operators in $W\left(x_{1}\right)$ is contracted with $F(x)$ and, hence, the corresponding momentum must not be on-shell. We have not worked out a general proof (in the style of appendix A) of the step from (104) to (105). But the explicit calculations show that this conclusion is correct. In Inserting (105) and (103) into (102) it results

$$
\left[Q, \mathcal{F}_{d}^{\mu \nu(1)}(x)\right]+i P_{d}^{\mu \nu}(x)-C \frac{1}{2}\left(f_{b c d}-f_{c b d}\right)\left[Q,: A_{b}^{\mu}(x) A_{c}^{\nu}(x):\right]=0,
$$

which means that the "local terms" must satisfy the condition (102) separately. Later when we shall know more about $W$ (which determines $P_{d}$ by

\footnotetext{
${ }^{44}$ We use here an additional normalization condition, namely that the term $\sim C \delta\left(x-x_{1}\right)$ : $A A$ : has the same color tensor as the corresponding non-local term (i.e. the term in $\Theta\left(x^{0}-x_{1}^{0}\right)\left[F^{\mu \nu}(x), W\left(x_{1}\right)\right]$ with the same external legs).

${ }^{45}$ Note that every operator valued distribution $G\left(x_{1}, \ldots, x_{n}\right),\left(x_{j} \in \mathbf{R}^{4}\right)$ can be written as divergence of a non-local operator, e.g. $G\left(x_{1}, \ldots, x_{n}\right)=\partial_{\nu}^{x_{1}} \int d y \partial^{\nu} D^{\cdots}\left(x_{1}-\right.$ y) $G\left(y, x_{2}, \ldots, x_{n}\right)$, where $D^{\cdots}$ is a fundamental solution of the wave equation.

${ }^{46}$ This remark concerns also the analogous (more complicated) steps from (110) to (113) and from (129) to (130) in second and third order.

${ }^{47}$ We set quotation marks because the splitting of a distribution in a local and a non-local part is non-unique.
} 
(105)), we will compute most of the coefficients in $\mathcal{F}_{d}^{(1)}(67)$ and the normalization constant $C$ from (106). Inserting (101) into (105) we find that $W$ must fulfill

$$
\left[F_{d}(x),\left[Q, W\left(x_{1}\right)\right]\right]=\partial_{\tau}^{x_{1}} R_{1 d}^{\tau}\left(x_{1}, x\right) \quad \text { for } \quad x_{1} \in\left(x+\bar{V}^{-}\right) \backslash\{x\} .
$$

Obviously this condition is truly weaker than $[Q, W]=$ (divergence of a Wick polynomial) (29). But the higher orders of the physicality requirement (69) yield more information about $W$.

- To second order (69) reads

$$
\begin{aligned}
& {\left[Q, \mathcal{F}_{d}^{(2)}(x)\right]+i \int d x_{1}\left[Q, R\left(W\left(x_{1}\right) ; \mathcal{F}_{d}^{(1)}(x)\right)\right]} \\
& \quad+\frac{i^{2}}{2} \int d x_{1} d x_{2}\left[Q, R\left(W\left(x_{1}\right) W\left(x_{2}\right) ; F_{d}(x)\right)\right]=0
\end{aligned}
$$

The tree diagrams in the third term have the structurets

$$
\begin{aligned}
& \left.R\left(W\left(x_{1}\right) W\left(x_{2}\right) ; F_{d}(x)\right)\right|_{\text {tree }} \\
= & \left.\sum \mathcal{D}_{1}^{\text {ret }}\left(x-x_{1}\right) \mathcal{D}_{2}^{\text {ret }}\left(x_{1}-x_{2}\right): B_{1}\left(x_{1}\right) B_{2}\left(x_{2}\right) B_{3}\left(x_{2}\right):+\left(x_{1} \leftrightarrow x_{(2}\right) 09\right)
\end{aligned}
$$

where $\mathcal{D}_{j}^{\text {ret }}=\Delta^{\text {ret }}, \partial \Delta^{\text {ret }},\left(\partial \partial \Delta^{\text {ret }}+C_{j} \delta\right)\left(C_{j}\right.$ are arbitrary constants $)$ and with free fields $B_{k}$ (possibly with derivative), i.e. $B_{k} \in\left\{A^{\mu}, \partial A, \partial \partial A,(\partial) u,(\partial) \tilde{u},(\partial) \phi,(\partial) H\right\}$. All other diagrams in (108) have less than three legs at the vertex/vertices $\neq x$. Now let $y_{l} \in \mathbf{R}^{4}, l=1,2,3$, with $\left(y_{l}-x\right)^{2}<0$ and let $\psi_{l}, l=1,2,3$, be arbitrary free fields. In the triple commutator of (108) with $\psi_{1}\left(y_{1}\right), \psi_{2}\left(y_{2}\right)$ and $\psi_{3}\left(y_{3}\right)$ only the terms (109) survive (the terms $\sim C_{1} \delta\left(x-x_{1}\right)$ in (109) do not contribute either)

$$
\begin{aligned}
0= & \int d x_{1} d x_{2}\left[\left[\left[\left[Q,\left.R\left(W\left(x_{1}\right) W\left(x_{2}\right) ; F_{d}(x)\right)\right|_{\text {tree }}\right], \psi_{1}\left(y_{1}\right)\right], \psi_{2}\left(y_{2}\right)\right], \psi_{3}\left(y_{3}\right)\right] \\
= & \int d x_{1} d x_{2}\left[\left[\left[\left[Q,\left\{\left[F_{d}(x), T\left(W\left(x_{1}\right) W\left(x_{2}\right)\right)\right]-W\left(x_{1}\right)\left[F_{d}(x), W\left(x_{2}\right)\right]\right.\right.\right.\right.\right. \\
& \left.\left.\left.\left.\left.-W\left(x_{2}\right)\left[F_{d}(x), W\left(x_{1}\right)\right]\right\}\left.\right|_{\text {tree }}\right], \psi_{1}\left(y_{1}\right)\right], \psi_{2}\left(y_{2}\right)\right], \psi_{3}\left(y_{3}\right)\right]
\end{aligned}
$$

where we have inserted (60), (61) and the causal factorization (4) of the time ordered products due to $x \notin\left\{x_{1}, x_{2}\right\}+\bar{V}^{-}$. Similarly to the step

\footnotetext{
${ }^{48}$ Note that totally retarded products (60) contain connected diagrams only.
} 
from (104) to (105) we conclude that $\left[Q,\left.R\left(W\left(x_{1}\right) W\left(x_{2}\right) ; F_{d}(x)\right)\right|_{\text {tree }}\right]$ must be a sum of divergences (with respect to $x_{1}$ or $x_{2}$ ) of local operators for $x_{1}, x_{2} \in\left(x+\bar{V}^{-}\right) \backslash\{x\}$. Now we transform (110) by means of identities of the kind

$$
\begin{aligned}
{\left[Q,\left.(M(x) N(y))\right|_{\text {tree }}\right] } & =\left.[Q, M(x) N(y)]\right|_{\text {tree }} \\
& =\left.([Q, M(x)] N(y))\right|_{\text {tree }}+\left.(M(x)[Q, N(y)])\right|_{\text {tre }}
\end{aligned}
$$

where $M$ and $N$ are arbitrary Wick polynomials. Then using (101) and (107) we find the condition

$$
\begin{aligned}
& {\left.\left[F_{d}(x),\left[Q, T\left(W\left(x_{1}\right) W\left(x_{2}\right)\right)\right]\right]\right|_{\text {tree }}-\left.\left[Q, W\left(x_{1}\right)\right]\left[F_{d}(x), W\left(x_{2}\right)\right]\right|_{\text {tree }}} \\
& \quad-\left.\left[Q, W\left(x_{2}\right)\right]\left[F_{d}(x), W\left(x_{1}\right)\right]\right|_{\text {tree }}=\operatorname{div}_{x_{1}}+\left(x_{1} \leftrightarrow x_{2}\right)
\end{aligned}
$$

for $x_{1}, x_{2} \in\left(x+\bar{V}^{-}\right) \backslash\{x\}$, where $\operatorname{div}_{y}$ means some divergence of a local operator with respect to $y$. Next we specialize to the subregion $x_{1}^{0}>x_{2}^{0}$, where $T\left(W\left(x_{1}\right) W\left(x_{2}\right)\right)$ factorizes. By means of again (107) we see that $W$ must satisfy

$$
\left.\left[\left[F_{d}(x), W\left(x_{1}\right)\right],\left[Q, W\left(x_{2}\right)\right]\right]\right|_{\text {tree }}=\operatorname{div}_{x_{1}}+\operatorname{div}_{x_{2}} \text { for } x_{1}, x_{2} \in\left(x+\bar{V}^{-}\right) \backslash\{x\}, x_{1}^{0}>x_{2}^{0} \text {. }
$$

Inserting the Ansatz for $W$ into this condition one finds that (114) yields the same restrictions on the parameters in $W$ as the $Q$-divergence condition to first order (29):

$$
[Q, W]=\text { divergence of a Wick polynomial. }
$$

In other words we obtain the same results for the parameters in $W$ as in (45), especially $f_{a b c}=\epsilon_{a b c}$. We recall that these values of the parameters are not only necessary for (115), they are also sufficient. Hence (107) is also fulfilled. By inserting (115) into (113) we find the condition

$$
\left[F_{d}(x),\left[Q,\left.T\left(W\left(x_{1}\right) W\left(x_{2}\right)\right)\right|_{\text {tree }}\right]\right]=\operatorname{div}_{x_{1}}+\left(x_{1} \leftrightarrow x_{2}\right) \quad \text { for } \quad x_{1}, x_{2} \in\left(x+\bar{V}^{-}\right) \backslash\{x\} .
$$

This is a necessary but not sufficient condition for $\left[Q,\left.T\left(W\left(x_{1}\right) W\left(x_{2}\right)\right)\right|_{\text {tree }}\right]=$ $\operatorname{div}_{x_{1}}+\left(x_{1} \leftrightarrow x_{2}\right)$. To get the full information of this latter condition we need to go to third order. 
- However, first we return to the "local terms" to first order (106), because we shall need the validity of this equation. We explicitly calculate the terms on the l.h.s. of (105), transform them into divergence form (as far as possible) and obtain"ty

$$
P_{d}^{\mu \nu}=\epsilon_{b c d}\left[F_{b}^{\mu \nu} u_{c}-A_{b}^{\mu} \partial^{\nu} u_{c}+A_{b}^{\nu} \partial^{\mu} u_{c}\right] .
$$

Inserting this into (106) we find that (106) is fulfilled iff the normalization

$$
C=-1
$$

is chosen and the first order coefficients in $\mathcal{F}_{d \text { int }}^{\mu \nu}$ take the values

$$
\begin{aligned}
& t_{d b c}^{(1)}=-\frac{1}{m_{c}} \epsilon_{b c d}, \quad \tilde{t}^{(1)}=0, \quad v^{(1)}=0, \quad \tilde{v}^{(1)}=0, \quad w^{(1)}=0, \quad \tilde{w}^{(1)}=0, \\
& w^{\prime(1)}=0, \quad \tilde{x}^{(1)}=0, \quad y^{(1)}=0, \quad \tilde{y}^{(1)}=0, \quad y^{\prime(1)}=0, \quad \tilde{z}^{(1)}=0(119)
\end{aligned}
$$

$x^{(1)}$ and $z^{(1)}$ are still arbitrary. Note that (107), (118) and (119) are not only necessary for (102), together they are also sufficient.

- We now consider the condition (69) to third order, i.e. the analogous equation to (102), (108). By commuting this equation with arbitrary free fields $\psi_{1}\left(y_{1}\right), \psi_{2}\left(y_{2}\right), \psi_{3}\left(y_{3}\right), \psi_{4}\left(y_{4}\right),\left(y_{l}-x\right)^{2}<0 \forall l$, we obtain

$$
\begin{array}{r}
0=\frac{i^{2}}{2 !} \int d x_{2} d x_{3}\left[\left[\left[\left[\left[Q, R\left(W\left(x_{2}\right) W\left(x_{3}\right) ; \mathcal{F}^{(1)}(x)\right)\right], \psi_{1}\left(y_{1}\right)\right], \psi_{2}\left(y_{2}\right)\right], \psi_{3}\left(y_{3}\right)\right], \psi_{4}\left(y_{4}\right)\right] \\
+\frac{i^{3}}{3 !} \int d x_{1} d x_{2} d x_{3}\left[\left[\left[\left[\left[Q, R\left(W\left(x_{1}\right) W\left(x_{2}\right) W\left(x_{3}\right) ; F(x)\right)\right], \psi_{1}\left(y_{1}\right)\right], \ldots \psi_{4}\left(y_{4}\right)\right\} .1\right.\right.
\end{array}
$$

Only tree diagrams of the following types contribute (we use the same notations as in (109)): in the first term

$\sum \mathcal{D}_{1}^{\mathrm{ret}}\left(x-x_{2}\right) \mathcal{D}_{2}^{\mathrm{ret}}\left(x-x_{3}\right): B_{0}(x) B_{1}\left(x_{2}\right) B_{2}\left(x_{2}\right) B_{3}\left(x_{3}\right) B_{4}\left(x_{3}\right):+\left(x_{2} \leftrightarrow x_{3}\right)$

(where $B_{0}(x)$ is a free field or $\equiv 1$ ), in the second term

$\sum \mathcal{D}_{3}^{\mathrm{ret}}\left(x-x_{1}\right) \mathcal{D}_{4}^{\mathrm{ret}}\left(x_{1}-x_{2}\right) \mathcal{D}_{5}^{\mathrm{ret}}\left(x_{1}-x_{3}\right): B_{5}\left(x_{2}\right) B_{6}\left(x_{2}\right) B_{7}\left(x_{3}\right) B_{8}\left(x_{3}\right):+\ldots$

\footnotetext{
${ }^{49} \mathrm{By}$ the experience of [2] we know that all contributions to $P_{d}$ come from the contraction of $F_{d}(x)$ with $\partial A\left(x_{1}\right)$ in the first term of $W\left(x_{1}\right)$ (41).
} 
and

$\sum \mathcal{D}_{6}^{\mathrm{ret}}\left(x-x_{1}\right) \mathcal{D}_{7}^{\mathrm{ret}}\left(x_{1}-x_{2}\right) \mathcal{D}_{8}^{\mathrm{ret}}\left(x_{2}-x_{3}\right): B_{9}\left(x_{1}\right) B_{10}\left(x_{2}\right) B_{11}\left(x_{3}\right) B_{12}\left(x_{3}\right):+\ldots$

where the dots mean terms obtained by cyclic permutations. In the first term in (120) we take (60) and the causal factorization basing on $x \notin\left(\left\{x_{2}, x_{3}\right\}+\right.$ $\left.\bar{V}^{-}\right)$into account. By means of (115) it results

$$
\begin{array}{r}
\frac{i^{2}}{2 !} \int d x_{2} d x_{3}\left[\left[\left[\left[\left\{\left[\left[Q, \mathcal{F}^{(1)}(x)\right], T\left(W\left(x_{2}\right) W\left(x_{3}\right)\right)\right]-W\left(x_{2}\right)\left[\left[Q, \mathcal{F}^{(1)}(x)\right], W\left(x_{3}\right)\right]\right.\right.\right.\right.\right. \\
\left.\left.\left.-W\left(x_{3}\right)\left[\left[Q, \mathcal{F}^{(1)}(x)\right], W\left(x_{2}\right)\right]\right\}\left.\right|_{\text {tree }}, \psi_{1}\left(y_{1}\right)\right] \ldots, \psi_{4}\left(y_{4}\right)\right](124)
\end{array}
$$

Additionally we have used that due to ([121) only the disconnected diagram of $T\left(W\left(x_{2}\right)\right.$

$\left.W\left(x_{3}\right)\right)$ contributes, hence $\left[\ldots\left[\mathcal{F}^{(1)}(x),\left[Q, T\left(W\left(x_{2}\right) W\left(x_{3}\right)\right)\right]\right] \ldots, \psi_{4}\left(y_{4}\right)\right]=\operatorname{div}_{x_{2}}+$ $\operatorname{div}_{x_{3}}$ by (115).

In the second term in (120) the situation is more complicated. The diagrams of the type (123) obey the causal factorization basing on $x \notin$ $\left(\left\{x_{1}, x_{2}, x_{3}\right\}+\bar{V}^{-}\right)$. But for the diagrams of the type (122) we only know $x \notin\left(\left\{x_{2}, x_{3}\right\}+\bar{V}^{-}\right)$(or promulgated configurations). To fix the position of the third vertex we consider two smooth functions $h_{1}, h_{2}$ with

$$
1=h_{1}(y)+h_{2}(y) \forall y \in x+\bar{V}^{-}, \quad h_{1} \in \mathcal{D}\left(\mathbf{R}^{4}\right), \quad x \in \operatorname{supp} h_{1},
$$

$\operatorname{supp} h_{2} \cap x+\bar{V}^{+}=\emptyset, \quad\left(y_{l}-z\right)^{2}<0 \forall z \in \operatorname{supp} h_{1}, l=1,2,3,4 .(125)$

With that the second term in $(\overline{120})$ can be written in the form

$$
\begin{aligned}
& \frac{i^{3}}{3 !} \int d x_{1} d x_{2} d x_{3}\left(h_{1}\left(x_{1}\right) h_{2}\left(x_{2}\right) h_{2}\left(x_{3}\right)+h_{2}\left(x_{1}\right) h_{1}\left(x_{2}\right) h_{2}\left(x_{3}\right)+h_{2}\left(x_{1}\right) h_{2}\left(x_{2}\right) h_{1}\left(x_{3}\right)\right. \\
& \left.+h_{2}\left(x_{1}\right) h_{2}\left(x_{2}\right) h_{2}\left(x_{3}\right)\right)\left[\ldots\left[Q,\left.R\left(W\left(x_{1}\right) \ldots ; F(x)\right)\right|_{\text {tree }}\right] \ldots, \psi_{4}\left(y_{4}\right)\right] .
\end{aligned}
$$

In the terms with a factor $h_{1}\left(x_{l}\right)$ the diagrams of the type (122) contribute only, and we may insert the causal factorization due to $\left\{x, x_{l}\right\} \cap\left(\left\{x_{j}, x_{k}\right\}+\right.$ $\left.\bar{V}^{-}\right)=\emptyset($ where $\{l, j, k\}=\{1,2,3\})$. So the contribution of $\left(h_{1}\left(x_{1}\right) h_{2}\left(x_{2}\right) h_{2}\left(x_{3}\right)+\right.$ $\left.h_{2}\left(x_{1}\right) h_{1}\left(x_{2}\right) h_{2}\left(x_{3}\right)+h_{2}\left(x_{1}\right) h_{2}\left(x_{2}\right) h_{1}\left(x_{3}\right)\right)$ in (126) reads

$$
\begin{aligned}
& \frac{i^{3}}{2 !} \int d x_{1} d x_{2} d x_{3} h_{1}\left(x_{1}\right) h_{2}\left(x_{2}\right) h_{2}\left(x_{3}\right)\left[\ldots \left\{\left[\left[Q, R\left(W\left(x_{1}\right) ; F(x)\right)\right], T\left(W\left(x_{2}\right) W\left(x_{3}\right)\right)\right]\right.\right. \\
& -W\left(x_{2}\right)\left[\left[Q, R\left(W\left(x_{1}\right) ; F(x)\right)\right], W\left(x_{3}\right)\right] \\
& \left.-W\left(x_{3}\right)\left[\left[Q, R\left(W\left(x_{1}\right) ; F(x)\right)\right], W\left(x_{2}\right)\right]\right\}\left.\right|_{\text {tree } \left.\ldots, \psi_{4}\left(y_{4}\right)\right]}
\end{aligned}
$$


where we have used $\left[Q, T\left(W\left(x_{2}\right) W\left(x_{3}\right)\right)\right]=\operatorname{div}_{x_{2}}+\operatorname{div}_{x_{3}}$, which holds true here for the same reason as in (124). ¿From (102) and (103) we know

$$
\begin{array}{r}
{\left[Q, \mathcal{F}^{(1)}(x)\right]+i \int d x_{1} h_{1}\left(x_{1}\right)\left[Q, R\left(W\left(x_{1}\right) ; F(x)\right)\right]=} \\
-i \int d x_{1} h_{2}\left(x_{1}\right)\left[Q, R\left(W\left(x_{1}\right) ; F(x)\right)\right]=-i \int d x_{1} h_{2}\left(x_{1}\right) \partial_{\tau}^{x_{1}}\left[F(x), W_{1}^{\tau}\left(x_{1}\right)\right](128
\end{array}
$$

where we have used $[Q, W]=\partial_{\tau} W_{1}^{\tau}$ (115). We now insert (127), (126) and (124) into $(120)$. By means of (128) we obtain

$$
\begin{aligned}
0= & \frac{i^{3}}{3 !} \int d x_{1} d x_{2} d x_{3} h_{2}\left(x_{1}\right) h_{2}\left(x_{2}\right) h_{2}\left(x_{3}\right)\left[\ldots \left\{\left[Q,\left.R\left(W\left(x_{1}\right) W\left(x_{2}\right) W\left(x_{3}\right) ; F(x)\right)\right|_{\text {tree }}\right]\right.\right. \\
& -\partial_{\tau}^{x_{1}}\left(\left[\left[F(x), W_{1}^{\tau}\left(x_{1}\right)\right], T\left(W\left(x_{2}\right) W\left(x_{3}\right)\right)\right]-W\left(x_{2}\right)\left[\left[F(x), W_{1}^{\tau}\left(x_{1}\right)\right], W\left(x_{3}\right)\right]\right. \\
& \left.\left.\left.-W\left(x_{3}\right)\left[\left[F(x), W_{1}^{\tau}\left(x_{1}\right)\right], W\left(x_{2}\right)\right]\right)\left.\right|_{\text {tree }}-\partial^{x_{2}}(\ldots)-\partial^{x_{3}}(\ldots)\right\} \ldots, \psi_{4}\left(y_{4}\right)\right], \quad(129)
\end{aligned}
$$

where $\partial^{x_{2}}(\ldots)$ and $\partial^{x_{3}}(\ldots)$ are obtained from $\partial^{x_{1}}(\ldots)$ by cyclic permutation. The next step is analogous to the step from (104) to (105), but more complicated because one has to care about the cancelation of the boundary terms. Taking the freedom in the choice of $h_{2}$ into account, (129) is equivalent to the existence of a local operator $R_{3}^{\tau}\left(x_{1} ; x_{2}, x_{3} ; x\right)$ with

$\left.\left[Q, R\left(W\left(x_{1}\right) W\left(x_{2}\right) W\left(x_{3}\right) ; F(x)\right)\right]\right|_{\text {tree }}=\partial_{\tau}^{x_{1}} R_{3}^{\tau}\left(x_{1} ; x_{2}, x_{3} ; x\right)+$ cyclic permutations

$$
\forall x_{1}, x_{2}, x_{3} \in\left(x+\bar{V}^{-}\right) \backslash\{x\}
$$

because the contribution from e.g. $\partial^{x_{1}}(\ldots)$ in $(\sqrt{129})$ is equal to

$$
-\frac{i^{3}}{3 !} \int d x_{1} d x_{2} d x_{3}\left(\partial_{\tau} h_{2}\right)\left(x_{1}\right) h_{2}\left(x_{2}\right) h_{2}\left(x_{3}\right)\left[\ldots R_{3}^{\tau}\left(x_{1} ; x_{2}, x_{3} ; x\right) \ldots, \psi_{4}\left(y_{4}\right)\right] .
$$

Let us explain this latter statement. First note that due to (62) and (125) there is only a contribution in (131) for $x_{1}, x_{2}, x_{3} \in\left(x+\bar{V}^{-}\right) \backslash\{x\} \wedge x_{1} \notin$ $\left(\left\{x_{2}, x_{3}\right\}+\bar{V}^{-}\right)$. In this region we have

$$
\begin{aligned}
R\left(W\left(x_{1}\right) W\left(x_{2}\right) W\left(x_{3}\right) ; F(x)\right)= & {\left[\left[F(x), W\left(x_{1}\right)\right], T\left(W\left(x_{2}\right) W\left(x_{3}\right)\right)\right] } \\
& -W\left(x_{2}\right)\left[\left[F(x), W\left(x_{1}\right)\right], W\left(x_{3}\right)\right] \\
& -W\left(x_{3}\right)\left[\left[F(x), W\left(x_{1}\right)\right], W\left(x_{2}\right)\right]
\end{aligned}
$$


due to causal factorization. Now we take into account that (in this region) $\partial_{\tau}^{x_{1}} R_{3}^{\tau}\left(x_{1}\right.$

$\left.x_{2}, x_{3} ; x\right)$ comes from the $\left[Q, W\left(x_{1}\right)\right]$-terms in $[Q, R(W W W ; F)]$ (where $R(W W W ; F)$ is given by (132)). However, by replacing $W\left(x_{1}\right)$ by $\partial_{\tau} W_{1}^{\tau}\left(x_{1}\right)$ in (132) we obtain exactly the $\partial^{x_{1}}(\ldots)$-term in $(129)$.

By means of causal factorization and (101), (115), (116) the condition (130) can be written in the form

$$
\begin{aligned}
& \left\{\left[F(x),\left[Q, T\left(W\left(x_{1}\right) W\left(x_{2}\right) W\left(x_{3}\right)\right)\right]\right]+\left[Q, T\left(W\left(x_{2}\right) W\left(x_{3}\right)\right)\right]\left[W\left(x_{1}\right), F(x)\right]\right. \\
& \left.+\left[Q, T\left(W\left(x_{1}\right) W\left(x_{2}\right)\right)\right]\left[W\left(x_{3}\right), F(x)\right]+\left[Q, T\left(W\left(x_{1}\right) W\left(x_{3}\right)\right)\right]\left[W\left(x_{2}\right), F(x)\right]\right\}\left.\right|_{\text {tree }} \\
= & \operatorname{div}_{x_{1}}+\text { cyclic permutations }
\end{aligned}
$$

for $x_{1}, x_{2}, x_{3} \in\left(x+\bar{V}^{-}\right) \backslash\{x\}$. Next we specialize to the subregion $x_{1} \notin$ $\left(\left\{x_{2}, x_{3}\right\}+\bar{V}^{-}\right)$. Again by causal factorization and (116) we find that $T\left(W\left(x_{2}\right) W\left(x_{3}\right)\right)$ must fulfill

$$
\left.\left[\left[F(x), W\left(x_{1}\right)\right],\left[Q, T\left(W\left(x_{2}\right) W\left(x_{3}\right)\right)\right]\right]\right|_{\text {tree }}=\operatorname{div}_{x_{1}}+\operatorname{div}_{x_{2}}+\operatorname{div}_{x_{3}}
$$

for $x_{1}, x_{2}, x_{3} \in\left(x+\bar{V}^{-}\right) \backslash\{x\} \wedge x_{1} \notin\left(\left\{x_{2}, x_{3}\right\}+\bar{V}^{-}\right)$. Inserting the explicit expression for $W$ (with the values of the parameters obtained so far) one sees that this condition is equivalent to

$$
\left[Q,\left.T\left(W\left(x_{2}\right) W\left(x_{3}\right)\right)\right|_{\text {tree }}\right]=\operatorname{div}_{x_{2}}+\left(x_{2} \leftrightarrow x_{3}\right)
$$

As we mentioned in section 2 the latter requirement is satisfied iff the masses agree $m \equiv m_{1}=m_{2}=m_{3}$ (47), the parameters of the $H$-coupling take the values (48) 50 , there is no term $\sim: H^{4}$ : in $W$ and if the normalization constants in the tree normalization terms (49) are suitably chosen (up to $\lambda$ (50) they are uniquely fixed by (135)). So far there remain two free parameters in $W$ and $\left.T(W W)\right|_{\text {tree }}: s$ (41) (cf. (48)) and $\lambda$ (50). In section 2 they have been determined by

$$
\left[Q,\left.T\left(W\left(x_{1}\right) W\left(x_{2}\right) W\left(x_{3}\right)\right)\right|_{\text {tree }}\right]=\operatorname{div}_{x_{1}}+\text { cyclic permutations. }
$$

By inserting (135) into (133) we find the weaker requirement

$$
\left[F(x),\left[Q,\left.T\left(W\left(x_{1}\right) W\left(x_{2}\right) W\left(x_{3}\right)\right)\right|_{\text {tree }}\right]\right]=\operatorname{div}_{x_{1}}+\text { cyclic permutations }
$$

\footnotetext{
${ }^{50}$ In the following calculations the undetermined $\operatorname{sign} \kappa$ in $(48)$ is chosen to be $\kappa=1$.
} 
for $x_{1}, x_{2}, x_{3} \in\left(x+\bar{V}^{-}\right) \backslash\{x\}$. But the latter condition does not determined $s$ and $\lambda$. One needs to consider the physicality condition (69) to fourth order. There the values (51) for $s$ and $\lambda$ are obtained, as can be seen by an analogous procedure. So we obtain exactly the same results for the parameters in $W$ and the tree normalizations in $T(W W)$ as in section 2. We recall that they agree completely with the interaction Lagrangian obtained by the Higgs mechanism.

- Now we have explained how to handle the essential difficulties which appear in the determination of the parameters in $W$ (41) and in $\mathcal{F}_{\text {int }}$ (67) by the physicality (69). We do not give further details. Instead we give a heuristic summary and the remaining results. To $n$-th order the requirement (69) reads

$$
0=\left[Q, \mathcal{F}_{d}^{(n)}(x)\right]+\sum_{l=1}^{n} \frac{i^{l}}{l !} \int d x_{1} \ldots d x_{l}\left[Q, R\left(W\left(x_{1}\right) \ldots W\left(x_{l}\right) ; \mathcal{F}_{d}^{(n-l)}(x)\right)\right]
$$

where $\mathcal{F}_{d}^{(0)} \equiv F_{d}$.

(I) In the "non-local terms" $\left(x_{j} \neq x\right.$ for at least one $\left.j\right)$ we can apply the causal factorization (4) of the time ordered products. Then the non-local terms in (138) cancel due to

$\left[Q, T\left(W\left(x_{1}\right) \ldots W\left(x_{k}\right)\right)\right]=$ sum of divergences of local operators, $\quad 1 \leq k \leq n$,

and the physicality (138) to lower orders $<n$ (see e.g. (128-129)). We have seen that for the tree diagrams to lowest orders the condition (139) is not only sufficient for the cancelation, it is also necessary. But the lowest orders tree diagrams of (139) fix the parameters in $W$ and $\left.T(W W)\right|_{\text {tree, as was shown }}$ in [2].

For the cancelation of the "non-local terms" in (138) it is not important which kind of physical fields we require to exist, the properties (i)-(vi) of $\mathcal{F}_{\text {int }}$ (67) are essentially not needed. Hence we conjecture that already the existence of any non-trivial observable fixes the interaction.

(II) The cancelation of the remaining terms in (138), which are "local" (i.e. their support is the total diagonal $x_{j}=x, \forall j$ ), requires a suitable normalization of the time ordered products $T\left(W\left(x_{1}\right) \ldots W\left(x_{l}\right) ; \mathcal{F}_{d}^{(n-l)}(x)\right)$ (see e.g. (118) ) and a suitable choice of the parameters in $\mathcal{F}_{d}^{(n-l)}, l=0,1, \ldots, n-$ 
1. In this way the latter parameters are uniquely determined (at least to lowest orders).

So the cancelation of the "local terms" to second order requires

$$
\begin{gathered}
t^{(2)}=0, \quad \tilde{t}^{(2)}=0, \quad v_{d a b c}^{(2)}=-\frac{1}{2 m^{2}}\left[\left(\delta_{d a}-\frac{m}{2} x_{d a}^{(1)}\right) \delta_{b c}-\frac{1}{2}\left(\delta_{d c} \delta_{b a}+\delta_{d b} \delta_{c a}\right)\right], \\
\tilde{v}^{(2)}=0, \quad w^{(2)}=0, \quad \tilde{w}^{(2)}=0, \quad w^{(2)}=0, \quad \tilde{x}^{(2)}=0, \\
y_{d b c}^{(2)}=\frac{1}{2 m^{2}} \epsilon_{d b c}-\frac{1}{m} x_{d a}^{(1)} \epsilon_{a b c}, \quad \tilde{y}^{(2)}=0, \quad y^{(2)}=0, \quad \tilde{z}^{(2)}=0, \quad z^{(1)}=\emptyset(140)
\end{gathered}
$$

The very last equation results from the terms $\sim g^{2}: F(x) u(x) H^{2}(x):$ In this case the cancelation is of a special kind as explained below. The other equations are obtained analogously to (106), (119). They come from bilinear and trilinear terms. $x^{(1)}, x^{(2)}$ and $z^{(2)}$ are still arbitrary.

To third order the terms $\sim g^{3}: F(x) u(x) \phi(x) \phi(x):,\left(\sim g^{3}: F(x) u(x) H^{2}(x)\right.$ : respectively) cancel iff

$$
x_{d a}^{(1)}=\frac{1}{m} \delta_{d a}, \quad\left(z_{d a}^{(2)}=\frac{1}{4 m^{2}} \delta_{d a} \text { respectively }\right) .
$$

Similarly one finds to fourth order that $x^{(2)}$ must vanish.

Summing up (119), (140) and (141) we obtain the result (70). But we have not checked that the cancelation of the "local terms" in (138) can be achieved for loop diagrams and for tree diagrams to higher orders. For the tree diagrams with more than three external legs (e.g. the very last equation in (140) and the terms considered in (141)) there is no contribution from $\mathcal{F}_{d}^{(n)}$ in (138) due to our Ansatz (67). Hence the parameters in $\mathcal{F}_{d}^{(n-l)}, l \geq 1$ are available only (besides normalization constants). However, most of the latter parameters have already been fixed (or restricted) by (138) to lower orders. (For example to achieve the cancelation of the terms considered in (141), it is important that $x^{(1)}\left(z^{(2)}\right.$ resp.) has not been fixed previously.) So we have shown the existence of observables $\mathcal{F}_{d \text { int }}^{\mu \nu}, d=1,2,3$, only partially. But there is a strong hint that this holds true for all terms to all orders from the identification (given in the main text) of our physical fields $\mathcal{F}_{d \text { int }}^{\mu \nu}(70)$ as gauge invariant fields in the framework of spontaneous symmetry breaking of the $S U(2)$ gauge symmetry.

\footnotetext{
${ }^{51}$ Mainly parameters in $\mathcal{F}_{d}^{(n)}$ are determined; most of the parameters in $\mathcal{F}_{d}^{(n-l)}, 1 \leq l \leq$ $n-1$, have already been fixed in earlier steps of the induction.
} 


\section{References}

[1] P. W. Anderson: 'Plasmons, Gauge Invariance, and Mass', Phys. Rev. 130, (1963) 439

[2] A. Aste, M. Dütsch and G. Scharf: 'On gauge invariance and spontaneous symmetry breaking', J. Phys. A, 30 (1997) 5785

M. Dütsch and G. Scharf: "Perturbative gauge invariance: the electroweak theory", Ann. Phys. (Leipzig) 8 (1999) 359

A. Aste, M. Dütsch and G. Scharf: "Perturbative gauge invariance: the electroweak theory II", Ann. Phys. (Leipzig) 8 (1999) 389

[3] C. Becchi, A. Rouet and R. Stora: "Renormalization of the abelian Higgs-Kibble model", Commun. Math. Phys. 42 (1975), 127

C. Becchi, A. Rouet and R. Stora: "Renormalization of gauge theories", Annals of Physics (N.Y.) 98 (1976), 287

[4] J. S. Bell: "High energy behavior of tree diagrams in gauge theories", Nucl. Phys. B60 (1973) 427-436

[5] N. N. Bogoliubov and D. V. Shirkov: "Introduction to the Theory of Quantized Fields", New York (1959)

[6] R. Brunetti and K. Fredenhagen: "Interacting quantum fields in curved space: Renormalization of $\phi^{4}$, gr-qc/9701048, Proceedings of the Conference 'Operator Algebras and Quantum Field Theory', held at Accademia Nazionale dei Lincei, Rome, July 1996

R. Brunetti and K. Fredenhagen: "Microlocal analysis and interacting quantum field theories: Renormalization on physical backgrounds", math-ph/9903028

[7] D. Buchholz and K. Fredenhagen: "A Note on the Inverse Scattering Problem in Quantum Field Theory", Commun. Math. Phys. 56 (1977), 91

[8] J.M. Cornwall, D. N. Levin and G. Tiktopoulos: "Derivation of gauge invariance from high-energy unitarity bounds on the $S$ matrix", Phys. Rev. D10 (1974) 1145 
[9] M. Dubois-Violette, private communication

[10] M. Dütsch and K. Fredenhagen: "A local (perturbative) construction of observables in gauge theories: the example of QED", hep-th/9807078, Commun. Math. Phys. 203 (1999) 71

[11] M. Dütsch, T. Hurth, F. Krahe and G. Scharf: "Causal construction of Yang-Mills theories. I." N. Cimento A 106 (1993), 1029

[12] M. Dütsch, T. Hurth and G. Scharf: "Causal construction of Yang-Mills theories. IV. Unitarity", N. Cimento A 108 (1995), 737

[13] M. Dütsch: "On gauge invariance of Yang-Mills theories with matter fields", N. Cimento A 109 (1996), 1145

[14] M. Dütsch: "Slavnov-Taylor identities from the causal point of view", Int. J. Mod. Phys. A 12 (1997) 3205

[15] M. Dütsch: "Non-Uniqueness of Quantized Yang-Mills Theories", J. Phys. A, 29 (1996) 7597

[16] M. Dütsch: "Causal Functional Integral for the S-Matrix of QED", N. Cim. A102 (1989) 1469

[17] H. Epstein and V. Glaser: "The role of locality in perturbation theory", Ann. Inst. H. Poincaré A 19 (1973), 211

[18] H. Epstein and V. Glaser: "Adiabatic limit in perturbation theory" in G.Velo and A.S.Wightman (eds.): Renormalization theory (1976) 193254

[19] L. D. Faddeev and V. N. Popov: "Feynman diagrams for the Yang-Mills field", Phys. Lett. B 25 (1967), 29

[20] D. R. Grigore: 'On the uniqueness of the non-abelian gauge theories in Epstein-Glaser approach to renormalization theory', hep-th/9806244

D. R. Grigore: The standard model and its generalizations in EpsteinGlaser approach to renormalization theory', hep-th/9810078

D. R. Grigore: The standard model and its generalizations in EpsteinGlaser approach to renormalization theory II: the fermion sector and the axial anomaly', hep-th/9903206 
[21] R. Haag, "Local Quantum Physics", Springer Verlag 1992

[22] S.S. Horuzhy, A.V. Voronin: "Remarks on mathematical structure of BRST theories",Commun. Math. Phys. 123 (1989), 677

[23] F. Krahe: "A causal approach to massive Yang-Mills theories", Acta Phys. Polonica B 27 (1996), 2453

[24] M. Karowski and P. Weisz: 'Exact form factors in $(1+1)$-dimensional field theoretic models with soliton behavior', Nucl. Phys. B 139, (1978) 455

[25] T. Kugo and I. Ojima: "Local covariant operator formalism of nonabelian gauge theories and quark confinement problem", Suppl. Progr. Theor. Phys. 66 (1979), 1

[26] C. H. Lewellyn Smith, in Proc. 5th Hawaii Topical Conf. Part. Phys., ed. P. N. Dobson et al., Univ. of Hawaii Press, Honolulu, Hawaii (1973)

[27] H. Reeh and S. Schlieder: "Bemerkungen zur Unitäräquivalenz von Lorentzinvarianten Feldern", N. Cimento 22 (1961), 1051

[28] M. Requardt: "Symmetry conservation and integrals over local charge densities in quantum field theory", Commun. Math. Phys. 50 (1976), 259

[29] J. J. Sakurai: 'Theory of Strong Interactions', Ann. Phys. (N.Y.) 11, (1960) 1

[30] G. Scharf: "General massive gauge theory", hep-th/9901140

[31] G. Scharf and M. Wellmann: "Quantum gravity from perturbative gauge invariance", hep-th/9903055

[32] B. Schroer and H. -W. Wiesbrock, "Modular Constructions of Quantum Field Theories with Interactions", hep-th/9812251, to be published in Rev. in Math. Phys.

[33] B. Schroer: 'Motivations and Physical Aims of Algebraic QFT', Ann. Phys. 255, No 3, (1997) 270 
[34] B. Schroer, "QFT at the Turn of the Century: old principles with new concepts" (an essay on local quantum physics), FU-SFB288 preprint, 1998

[35] B. Schroer, "Modular Wedge Localzation and the $\mathrm{d}=1+1$ Formfactor Program", Annals of Physics 275, No 2 (1999) 190

[36] B. Schroer: "New Concepts in Particle Physics from Solution of an Old Problem", hep-th/9908021

[37] Schweber, "An Introduction to Relativistic Quantum Field Theory", Part Four, Row, Peterson and Company 1961

[38] J. Schwinger: 'Gauge Invariance and Mass', Phys. Rev. 125, (1962) 397

[39] F. A. Smirnov: "Formfactors in completely integrable Models of Quantum Field Theory", Adv. Series in Math. Phys. 14, World Scientific 1992

[40] O. Steinmann, "Perturbation expansions in axiomatic field theory", Lecture Notes in Physics 11, Springer-Verlag (1971)

[41] R. Stora: "Local gauge groups in quantum field theory: perturbative gauge theories", talk given at the workshop "Local Quantum Physics" at the Erwin-Schroedinger-Institute, Vienna (1997)

[42] R. F. Streater and A.S. Wightman, "TCP, Spin \& Statistics and All That"

[43] J. A. Swieca, 'Charge screening and mass spectrum', Phys. Rev. D 13, (1976) 312

[44] G. 't Hooft, "The glorious days of physics, Renormalization of Gauge Theories" hep-th/9812203

[45] G. 't Hooft and M. T. Veltman, "Combinatorics of Gauge Fields", Nucl. Phys. B 50, (1972) 318

[46] S. Weinberg: "The Theory of Quantized Fields", Vol.I 